\title{
Machine Learning-Based Early Warning Systems for Clinical Deterioration: Systematic Scoping Review
}

Sankavi Muralitharan ${ }^{1,2}$, MPharm, MSc; Walter Nelson $^{1 *}$, BSc; Shuang Di $^{1,3^{*}}$, BSc, MEd, MSc; Michael McGillion ${ }^{4,5}$, BScN, PhD; PJ Devereaux ${ }^{5,6}$, MD, PhD, FRCPC; Neil Grant Barr ${ }^{7}$, BA, MSc, PhD; Jeremy Petch ${ }^{1,5,8,}$, HBA, MA, $\mathrm{PhD}$

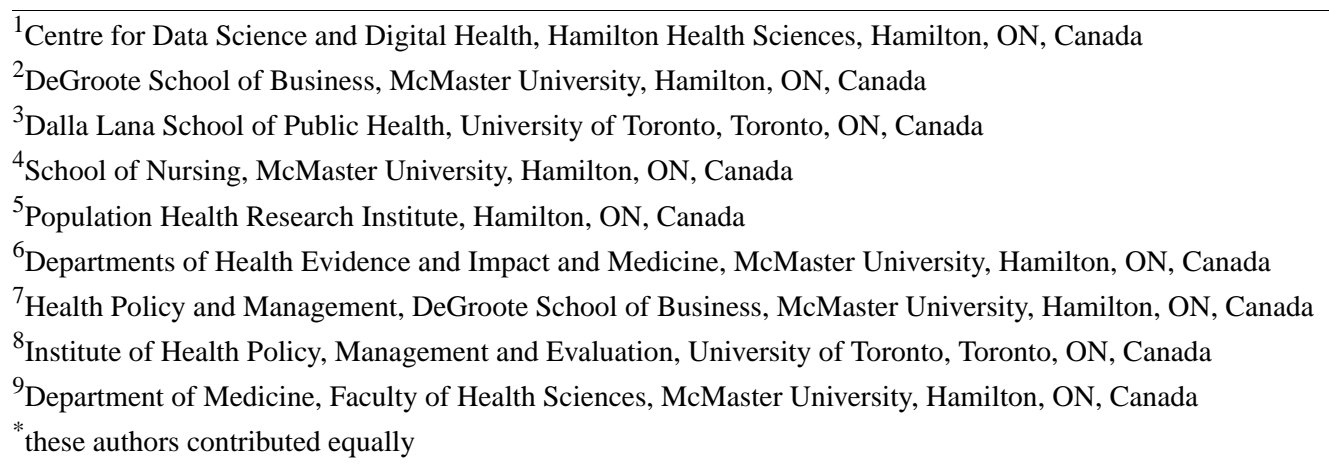

Corresponding Author:

Sankavi Muralitharan, MPharm, MSc

Centre for Data Science and Digital Health

Hamilton Health Sciences

293 Wellington St. N

Hamilton, ON, L8L 8E7

Canada

Phone: 12897882965

Email: sankavi22@hotmail.com

\section{Abstract}

Background: Timely identification of patients at a high risk of clinical deterioration is key to prioritizing care, allocating resources effectively, and preventing adverse outcomes. Vital signs-based, aggregate-weighted early warning systems are commonly used to predict the risk of outcomes related to cardiorespiratory instability and sepsis, which are strong predictors of poor outcomes and mortality. Machine learning models, which can incorporate trends and capture relationships among parameters that aggregate-weighted models cannot, have recently been showing promising results.

Objective: This study aimed to identify, summarize, and evaluate the available research, current state of utility, and challenges with machine learning-based early warning systems using vital signs to predict the risk of physiological deterioration in acutely ill patients, across acute and ambulatory care settings.

Methods: PubMed, CINAHL, Cochrane Library, Web of Science, Embase, and Google Scholar were searched for peer-reviewed, original studies with keywords related to "vital signs," "clinical deterioration," and "machine learning." Included studies used patient vital signs along with demographics and described a machine learning model for predicting an outcome in acute and ambulatory care settings. Data were extracted following PRISMA, TRIPOD, and Cochrane Collaboration guidelines.

Results: We identified 24 peer-reviewed studies from 417 articles for inclusion; 23 studies were retrospective, while 1 was prospective in nature. Care settings included general wards, intensive care units, emergency departments, step-down units, medical assessment units, postanesthetic wards, and home care. Machine learning models including logistic regression, tree-based methods, kernel-based methods, and neural networks were most commonly used to predict the risk of deterioration. The area under the curve for models ranged from 0.57 to 0.97 .

Conclusions: In studies that compared performance, reported results suggest that machine learning-based early warning systems can achieve greater accuracy than aggregate-weighted early warning systems but several areas for further research were identified. While these models have the potential to provide clinical decision support, there is a need for standardized outcome measures to 
allow for rigorous evaluation of performance across models. Further research needs to address the interpretability of model outputs by clinicians, clinical efficacy of these systems through prospective study design, and their potential impact in different clinical settings.

(J Med Internet Res 2021;23(2):e25187) doi: 10.2196/25187

\section{KEYWORDS}

machine learning; early warning systems; clinical deterioration; ambulatory care; acute care; remote patient monitoring; vital signs; sepsis; cardiorespiratory instability; risk prediction

\section{Introduction}

Patient deterioration and adverse outcomes are often preceded by abnormal vital signs [1-3]. These warning signs frequently appear a few hours to a few days before the event, which can provide sufficient time for intervention. In response, clinical decision support early warning systems (EWS) have been developed that employ periodic observations of vital signs along with a predetermined criteria or cut-off range for alerting clinicians of patient deterioration [4].

EWS typically employ heart rate (HR), respiratory rate (RR), blood pressure (BP), peripheral oxygen saturation $\left(\mathrm{SpO}_{2}\right)$, temperature, and sometimes the level of consciousness [5]. Aggregate-weighted EWS incorporate several vital signs and other patient characteristics with clearly defined thresholds. Weights are assigned to each of these vital signs and characteristics based on a threshold, and an overall risk score is calculated by adding each of the weighted scores [6].

Some of the commonly used aggregate-weighted EWS for predicting cardiorespiratory insufficiency and mortality are the Modified Early Warning Score (MEWS) [7], National Early Warning Score (NEWS) [8], and Hamilton Early Warning Score [9], which all incorporate vital signs and the level of consciousness (Alert, Verbal, Pain, Unresponsive [AVPU]) but have varying thresholds for assigning scores.

The predictive ability of aggregate-weighted EWS has limitations. First, the scores indicate the present risk of the patient but do not incorporate trends nor provide information about the possible risk trajectory [6]; thus, the scores do not communicate whether the patient is improving or deteriorating and the rate of this change [10]. Second, these scores do not capture any correlations between the parameters, as the score for each parameter is calculated independently through simple addition [6] (eg, HR or RR can be interpreted differently when body temperature is taken into consideration).

A newer approach to EWS relies on machine learning (ML). ML models learn patterns and relationships directly from data rather than relying on a rule-based system [11]. Unlike aggregate-weighted EWS, ML models are computationally intensive, but can incorporate trends in risk scores, adjust for varying numbers of clinical covariates, and be optimized for different care settings and populations [12]. Like other EWS, ML models can be integrated into electronic health records to analyze vital sign measurements continuously and provide predictions of patient outcomes as part of a clinical decision support system [13].
Two systematic reviews in 2019 [14,15] evaluated the ability of ML models to predict clinical deterioration in adult patients using vital signs. The review by Brekke et al [15] examined the utility of trends within intermittent vital sign measurements from adult patients admitted to all hospital wards and emergency departments (ED) but identified only 2 retrospective studies that met their inclusion criteria. The review identified that vital sign trends were of value in detecting clinical deterioration but concluded that there is a lack of research in intermittently monitored vital sign trends and highlighted the need for controlled trials.

The review conducted by Linnen et al [14] compared the accuracy and workload of ML-based EWS with that of aggregate-weighted EWS. This review focused on studies that reported adult patient transfers to intensive care units (ICUs) or mortality as the outcome(s) and excluded all other clinical settings; 6 studies were identified that reported the performance metrics for both the ML-based EWS and aggregate-weighted EWS. The review identified that ML modelling consistently performed better than aggregate-weighted models while generating clinical workload. They also highlighted the need for standardized performance metrics and deterioration outcome definitions.

These are important findings, but to date no review has systematically reviewed the evidence from studies using ML-based EWS using vital sign measurements of varying frequencies, across different care settings and clinical outcomes in order to identify common methodological trends and limitations with current approaches to generate recommendations for future research in this area.

The objective of this study was to scope the state of research in ML-based EWS using vital signs data for predicting the risk of physiological deterioration in patients across acute and ambulatory care settings and to identify directions for future research in this area.

\section{Methods}

A systematic scoping review was conducted by following the Preferred Reporting Items for Systematic Reviews and Meta-Analyses (PRISMA) extension for scoping reviews (PRISMA-ScR) framework [16]. This process provides an analysis of the available research, current state of utility of ML-based EWS, challenges facing their clinical implementation, and how they compare to aggregate-weighted EWS by identifying, synthesizing, and appraising the relevant evidence in the area. The literature search, assessment of eligibility of 
full-text articles, inclusion in the review, and extraction of study data were carried out by a single author.

\section{Search Strategy}

We searched PubMed, CINAHL, Cochrane Library, Web of Science, Embase, and Google Scholar for peer-reviewed studies without using any filters for study design and language. Searches were also conducted without any date restrictions. The reference lists of all studies that met the inclusion criteria were screened for additional articles. The search strategy involved a series of searches using a combination of relevant keywords and synonyms, including "vital signs," "clinical deterioration," and "machine learning." See Multimedia Appendix 1 for search terms.

\section{Eligibility Criteria}

The inclusion criteria covered the following:

- Peer-reviewed studies evaluating continuous or intermittent vital sign monitoring in adult patients so that all data collection or sampling frequencies (eg, 1 measurement per minute vs 1 measurement every 2 hours) wedre taken into consideration;

- Studies conducted using data gathered from all acute and ambulatory care settings including medical or surgical hospital wards, ICUs, step-down units, ED, and in-home care;

- Quantitative, observational, retrospective, and prospective cohort studies and randomized controlled trials;

- Studies that involved ML or multivariable statistical or ML models and reported some model performance measure (eg, area under the curve) [17];

- Studies that reported mortality or any outcomes related to clinical deterioration so that EWS models and performance can be examined for all explored outcomes.

The exclusion criteria included the following:

- Studies that used any laboratory values as predictors for the ML-based EWS, as this review focuses on examining time-sensitive predictions of clinical deterioration using patient parameters that are readily available across all care settings;

- Studies involving pediatric or obstetric populations due to these patients having different or altered physiologies that cannot be compared to standard adult patients;
- Qualitative studies, reviews, preprints, case reports, commentaries, or conference proceedings.

\section{Study Selection}

References from the preliminary searches were handled using Mendeley reference management software. After duplicates were removed, titles and abstracts were screened to assess preliminary eligibility. Eligible studies were then read in full length to be assessed against the inclusion and exclusion criteria.

\section{Data Extraction}

Data were extracted from eligible studies using an extraction sheet that followed the PRISMA [18] and Cochrane Collaboration guidelines for systematic reviews [19] and the Transparent Reporting of a Multivariable Prediction Model for Individual Prognosis or Diagnosis (TRIPOD) guidelines [20] for the reporting of predictive models. Study characteristics, setting, demographics, patient outcomes, ML model characteristics, and model performance data were extracted. The model performance results were extracted from the validation data set rather than from the model derivation or training data set to decrease the potential for model overfitting. When studies explored multiple ML models, the model with the best performance was selected for reporting and comparison. If studies compared the performance of ML models to aggregate-weighted EWS, then the performance data of these warning systems were also extracted.

\section{Results}

\section{Search Results and Study Selection}

The search for "vital signs" AND "clinical deterioration" AND "machine learning" using the same query terms and filters identified 417 studies after duplicate removal. During the title and abstract screening process, 386 studies were excluded. Of the 31 full-text articles that were assessed, 7 studies were excluded for not meeting the eligibility criteria: 2 studies did not use ML models to predict deterioration, 3 studies included vital sign measurements in addition to laboratory values as predictors, 1 study focused on a cohort of pregnant women, and 1 study did not meet the criteria for model performance measures. A review of the reference lists of the 24 selected studies did not yield any additional studies fulfilling the eligibility criteria (refer to Figure 1). 
Figure 1. PRISMA flowchart of the search strategy and study selection.

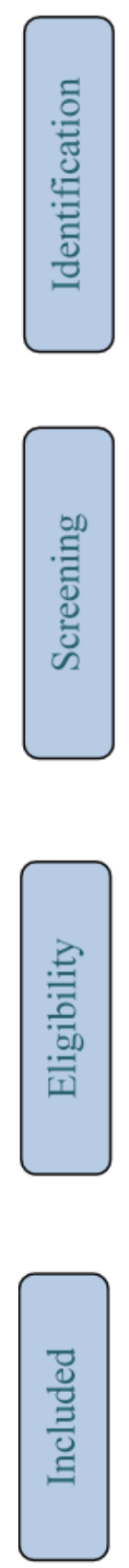

\section{Study Characteristics}

Of the selected studies, 23 conducted a retrospective analysis of the vital signs data, while 1 study [21] used a prospective cohort study design. Seventeen studies only analyzed continuous vital signs measurements collected through wearable devices and bedside monitors, whereas 3 [22-24] studies analyzed vital signs that were collected both manually and intermittently by clinical staff. Two studies $[25,26]$ analyzed vital signs that were collected both continuously and intermittently, while the remaining 2 studies did not report how the vital sign data were collected.
Studies included in scoping review $(n=24)$

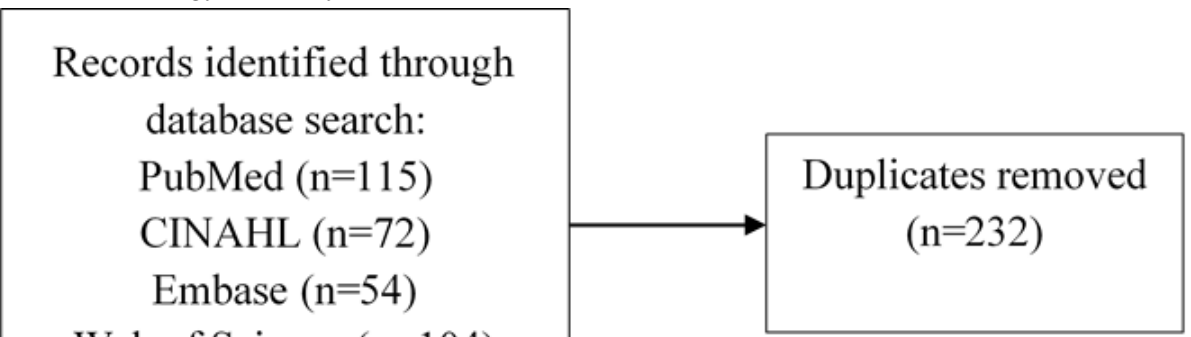

Web of Science $(n=104)$

Cochrane Library $(\mathrm{n}=0)$

Google Scholar $(n=304)$

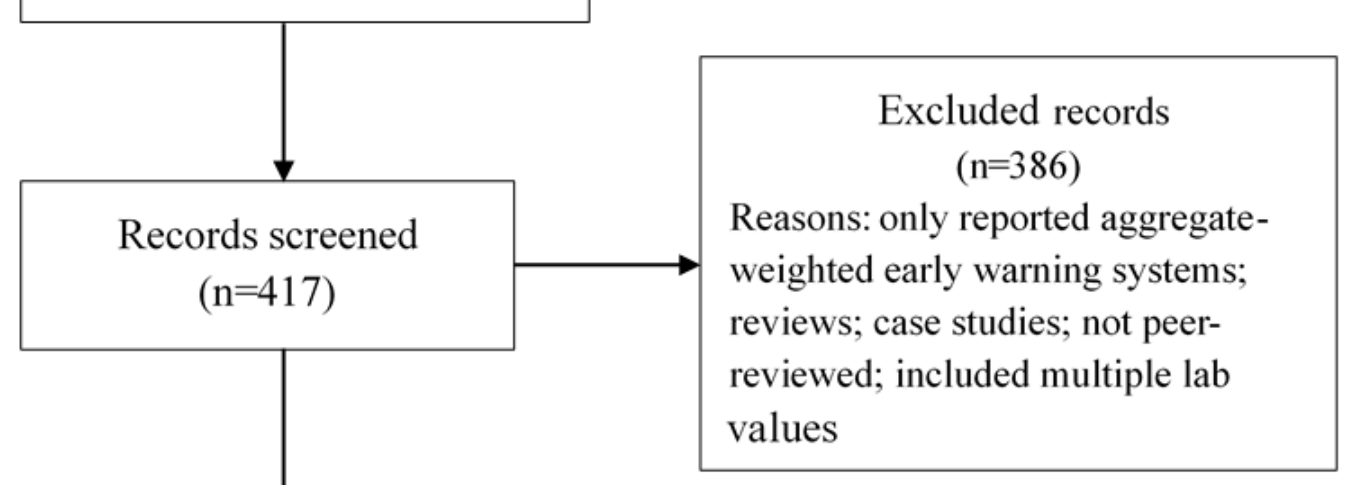

Full-text articles excluded

No machine learning models $(\mathrm{n}=2)$

Included lab values and vitals $(n=3)$

Pregnant cohort $(\mathrm{n}=1)$

No model performance measure $(n=1)$
Studies were conducted in a variety of settings within hospitals while the study by Larburu et al [22] was conducted in an ambulatory setting. While 3 studies [27-29] aimed to develop a remote home-based monitoring tool, the vital sign data used were obtained from the Medical Information Mart for Intensive Care (MIMIC and MIMIC-II) databases [30,31] consisting of data captured from patient monitors in different ICUs. Regarding location, 5 studies [24,26,32-34] were conducted on general wards, 4 studies $[11,23,35,36]$ were conducted in EDs, 7 studies [26,34,37-41] were conducted in ICUs, 2 studies [25,42] were conducted in postoperative wards, and 4 studies [21,43-45] in acute stay wards (medical admission unit, step-down units). Cohort sizes for the studies ranged from 12 patients [39] to $10,967,518$ patient visits [11] (refer to Table 1 ). 
Table 1. Study characteristics.

\begin{tabular}{|c|c|c|c|c|c|c|c|c|}
\hline $\begin{array}{l}\text { Authors, } \\
\text { year }\end{array}$ & Setting(s) & $\begin{array}{l}\text { Data collec- } \\
\text { tion }\end{array}$ & $\begin{array}{l}\text { Cohort descrip- } \\
\text { tion }\end{array}$ & Event rate & Study purpose & Predictors & $\begin{array}{l}\text { Measurement } \\
\text { frequency }\end{array}$ & Outcome \\
\hline $\begin{array}{l}\text { Badriyah et } \\
\text { al, } 2014 \\
{[45]}\end{array}$ & $\begin{array}{l}\text { Medical as- } \\
\text { sessment } \\
\text { unit for } 24 \\
\text { hours }\end{array}$ & $\begin{array}{l}\text { Personal digi- } \\
\text { tal assistants } \\
\text { running Vital- } \\
\text { PAC software }\end{array}$ & $\begin{array}{l}35,585 \text { admis- } \\
\text { sions }\end{array}$ & $\begin{array}{l}199(0.56 \%), \\
\text { cardiac arrest; } \\
1161(3.26 \%) \\
\text { unanticipated } \\
\text { ICU }^{\mathrm{a}} \text { admis- } \\
\text { sions; } 1789 \\
(5.02 \%) \text { deaths; } \\
3149(8.85 \%) \\
\text { any outcome }\end{array}$ & $\begin{array}{l}\text { Compare the per- } \\
\text { formance of a de- } \\
\text { cision tree analy- } \\
\text { sis with NEWS }\end{array}$ & $\begin{array}{l}\mathrm{HR}^{\mathrm{c}}, \mathrm{RR}^{\mathrm{d}}, \\
\mathrm{SBP}^{\mathrm{e}}, \text { tempera- } \\
\text { ture, } \mathrm{SpO}_{2}, \\
\mathrm{AVPU}^{\mathrm{f}} \text { level, } \\
\% \text { breathing } \\
\text { air at the time } \\
\text { of } \mathrm{SpO}_{2} \text { mea- } \\
\text { surement }\end{array}$ & Not specified & $\begin{array}{l}\text { Cardiac arrest, } \\
\text { unanticipated } \\
\text { ICU admission, } \\
\text { or death, each } \\
\text { within } 24 \text { hours } \\
\text { of a given vital } \\
\text { sign observa- } \\
\text { tion }\end{array}$ \\
\hline $\begin{array}{l}\text { Chen at al, } \\
2017 \text { [44] }\end{array}$ & $\begin{array}{l}\text { Step-down } \\
\text { unit }\end{array}$ & $\begin{array}{l}\text { Bedside moni- } \\
\text { tors }\end{array}$ & $\begin{array}{l}1880 \text { patients } \\
(1971 \text { admis- } \\
\text { sions) }\end{array}$ & $\begin{array}{l}997 \text { patients } \\
(53 \%) \text { or } 1056 \\
\text { admissions } \\
(53.6 \%) \text { who } \\
\text { experienced } \\
\text { CRI }^{\text {g events }}\end{array}$ & $\begin{array}{l}\text { Describe the dy- } \\
\text { namic and person- } \\
\text { al character of } \\
\text { CRI risk evolu- } \\
\text { tion observed } \\
\text { through continu- } \\
\text { ous vital sign } \\
\text { monitoring of in- } \\
\text { dividual patients }\end{array}$ & $\begin{array}{l}\mathrm{HR}, \mathrm{RR}, \\
\mathrm{SPO}_{2} \text { (at } 1 / 20 \\
\mathrm{~Hz}), \mathrm{SBP} \\
\mathrm{DBP}^{\mathrm{h}}\end{array}$ & Every 2 hours & CRI \\
\hline $\begin{array}{l}\text { Churpek et } \\
\text { al, } 2016 \\
{[24]}\end{array}$ & $\begin{array}{l}\text { All wards at } \\
\text { the Universi- } \\
\text { ty of Chica- } \\
\text { go and } 4 \\
\text { North Shore } \\
\text { University } \\
\text { Health Sys- } \\
\text { tem hospitals }\end{array}$ & $\begin{array}{l}\text { Data collected } \\
\text { manually, doc- } \\
\text { umented elec- } \\
\text { tronically }\end{array}$ & $\begin{array}{l}269,999 \text { admis- } \\
\text { sions }\end{array}$ & $\begin{array}{l}16,452 \text { out- } \\
\text { comes }(6.09 \%)\end{array}$ & $\begin{array}{l}\text { Whether adding } \\
\text { trends improves } \\
\text { accuracy of early } \\
\text { detection of clini- } \\
\text { cal deterioration } \\
\text { and which meth- } \\
\text { ods are optimal } \\
\text { for modelling } \\
\text { trends }\end{array}$ & $\begin{array}{l}\text { Temperature, } \\
\mathrm{HR}, \mathrm{RR}, \\
\mathrm{SpO}_{2}, \mathrm{DBP}, \\
\mathrm{SBP}\end{array}$ & Every 4 hours & $\begin{array}{l}\text { Development of } \\
\text { critical illness } \\
\text { on the wards: } \\
\text { deaths, cardiac } \\
\text { arrest, ICU } \\
\text { transfers }\end{array}$ \\
\hline $\begin{array}{l}\text { Chiu et al, } \\
2019 \text { [42] }\end{array}$ & $\begin{array}{l}\text { Postopera- } \\
\text { tive surgical } \\
\text { wards at } 4 \\
\text { UK adult } \\
\text { cardiac surgi- } \\
\text { cal centers }\end{array}$ & $\begin{array}{l}\text { VitalPac to } \\
\text { electronically } \\
\text { capture pa- } \\
\text { tients' vital } \\
\text { signs }\end{array}$ & $\begin{array}{l}\text { Adults under- } \\
\text { going risk- } \\
\text { stratified ma- } \\
\text { jor cardiac } \\
\text { surgery, } \\
n=13,631\end{array}$ & $\begin{array}{l}578 \text { patients } \\
(4.2 \%) \text { with an } \\
\text { outcome; } 499 \\
\text { patients } \\
(3.66 \%) \text { with } \\
\text { unplanned ICU } \\
\text { readmissions }\end{array}$ & $\begin{array}{l}\text { Using logistic re- } \\
\text { gression to model } \\
\text { the association of } \\
\text { NEWS variables } \\
\text { with a serious pa- } \\
\text { tient event in the } \\
\text { subsequent } 24 \\
\text { hours; secondary } \\
\text { objectives: com- } \\
\text { paring the dis- } \\
\text { criminatory pow- } \\
\text { er of each model } \\
\text { for events in the } \\
\text { next } 6 \text { hours or } \\
12 \text { hours }\end{array}$ & $\begin{array}{l}\mathrm{RR}, \mathrm{SpO}_{2}, \\
\mathrm{SBP}, \mathrm{HR}, \\
\text { temperature, } \\
\text { consciousness } \\
\text { level }\end{array}$ & Not specified & $\begin{array}{l}\text { Death, cardiac } \\
\text { arrest, un- } \\
\text { planned ICU } \\
\text { readmissions }\end{array}$ \\
\hline $\begin{array}{l}\text { Clifton et } \\
\text { al, } 2014 \\
{[25]}\end{array}$ & $\begin{array}{l}\text { Postopera- } \\
\text { tive ward of } \\
\text { the cancer } \\
\text { center, Ox- } \\
\text { ford Univer- } \\
\text { sity Hospi- } \\
\text { tals NHS } \\
\text { Trust, Unit- } \\
\text { ed Kingdom }\end{array}$ & $\begin{array}{l}\text { Continuous vi- } \\
\text { tals monitored } \\
\text { by wearable } \\
\text { devices; inter- } \\
\text { mittent vitals } \\
\text { monitored } \\
\text { manually by } \\
\text { ward staff }\end{array}$ & $\begin{array}{l}200 \text { patients in } \\
\text { the postopera- } \\
\text { tive ward fol- } \\
\text { lowing upper } \\
\text { gastrointesti- } \\
\text { nal cancer } \\
\text { surgery }\end{array}$ & Not specified & $\begin{array}{l}\text { Using continuous } \\
\text { vitals monitoring } \\
\text { to provide early } \\
\text { warning of physi- } \\
\text { ological deteriora- } \\
\text { tion, such that } \\
\text { preventative clini- } \\
\text { cal action may be } \\
\text { taken }\end{array}$ & $\begin{array}{l}\mathrm{SpO}_{2}, \mathrm{HR} \\
(256 \mathrm{~Hz}), \mathrm{BP}, \\
\mathrm{RR}\end{array}$ & $\begin{array}{l}\text { Continuously } \\
\left(\mathrm{SpO}_{2}, \mathrm{HR}\right) \text {, } \\
\text { intermittently } \\
(\mathrm{BP}, \mathrm{RR})\end{array}$ & $\begin{array}{l}\text { Physiological } \\
\text { deterioration }\end{array}$ \\
\hline
\end{tabular}




\begin{tabular}{|c|c|c|c|c|c|c|c|c|}
\hline $\begin{array}{l}\text { Authors, } \\
\text { year }\end{array}$ & Setting(s) & $\begin{array}{l}\text { Data collec- } \\
\text { tion }\end{array}$ & $\begin{array}{l}\text { Cohort descrip- } \\
\text { tion }\end{array}$ & Event rate & Study purpose & Predictors & $\begin{array}{l}\text { Measurement } \\
\text { frequency }\end{array}$ & Outcome \\
\hline $\begin{array}{l}\text { Desautels } \\
\text { et al, } 2016 \\
\text { [37] }\end{array}$ & $\begin{array}{l}\text { Beth Israel } \\
\text { Deaconess } \\
\text { Medical } \\
\text { Center ICU }\end{array}$ & $\begin{array}{l}\text { ICU bedside } \\
\text { monitors and } \\
\text { medical } \\
\text { records (MIM- } \\
\left.\mathrm{IC}^{\mathrm{m}}-\mathrm{III}\right)\end{array}$ & $\begin{array}{l}22,853 \mathrm{ICU} \\
\text { stays }\end{array}$ & $\begin{array}{l}2577(11.28 \%) \\
\text { stays with con- } \\
\text { firmed sepsis }\end{array}$ & $\begin{array}{l}\text { Validate a sepsis } \\
\text { prediction } \\
\text { method, InSight, } \\
\text { for the new Sep- } \\
\text { sis-3 definitions } \\
\text { and make predic- } \\
\text { tions using a min- } \\
\text { imal set of vari- } \\
\text { ables }\end{array}$ & $\begin{array}{l}\mathrm{GCS}, \mathrm{HR}, \mathrm{RR}, \\
\mathrm{SpO}_{2} \text {, temper- } \\
\text { ature, invasive } \\
\text { and noninva- } \\
\text { sive SBP and } \\
\text { DBP }\end{array}$ & $\begin{array}{l}\text { At least } 1 \\
\text { measurement } \\
\text { per hour }\end{array}$ & Onset of sepsis \\
\hline $\begin{array}{l}\text { Forkan et } \\
\text { al, } 2017 \\
{[28]}\end{array}$ & $\begin{array}{l}\text { Beth Israel } \\
\text { Deaconess } \\
\text { Medical } \\
\text { Center ICU }\end{array}$ & $\begin{array}{l}\text { ICU bedside } \\
\text { monitors and } \\
\text { medical } \\
\text { records (MIM- } \\
\text { IC-II) }\end{array}$ & 1023 patients & Not specified & $\begin{array}{l}\text { Develop a proba- } \\
\text { bilistic model for } \\
\text { predicting the fu- } \\
\text { ture clinical } \\
\text { episodes of a pa- } \\
\text { tient using ob- } \\
\text { served vital sign } \\
\text { values prior to } \\
\text { the clinical event }\end{array}$ & $\begin{array}{l}\mathrm{HR}, \mathrm{SBP}, \\
\mathrm{DBP}, \text { mean } \\
\mathrm{BP}, \mathrm{RR}, \mathrm{SpO}_{2}\end{array}$ & $\begin{array}{l}\text { All samples } \\
\text { converted to } \\
\text { per-minute } \\
\text { sampling }\end{array}$ & $\begin{array}{l}\text { Abnormal clini- } \\
\text { cal events }\end{array}$ \\
\hline $\begin{array}{l}\text { Forkan et } \\
\text { al, } 2017 \\
{[27]}\end{array}$ & $\begin{array}{l}\text { Beth Israel } \\
\text { Deaconess } \\
\text { Medical } \\
\text { Center ICU }\end{array}$ & $\begin{array}{l}\text { ICU bedside } \\
\text { monitors and } \\
\text { medical } \\
\text { records (MIM- } \\
\text { IC \& MIMIC- } \\
\text { II) }\end{array}$ & 85 patients & Not specified & $\begin{array}{l}\text { Develop an intel- } \\
\text { ligent method for } \\
\text { personalized } \\
\text { monitoring and } \\
\text { clinical decision } \\
\text { support through } \\
\text { early estimation } \\
\text { of patient-specif- } \\
\text { ic vital sign val- } \\
\text { ues }\end{array}$ & $\begin{array}{l}\mathrm{HR}, \mathrm{SBP}, \\
\mathrm{DBP}, \text { mean } \\
\mathrm{BP}, \mathrm{RR}, \mathrm{SpO}_{2}\end{array}$ & $\begin{array}{l}\text { Per-minute } \\
\text { sampling }\end{array}$ & $\begin{array}{l}\text { Patient-specific } \\
\text { anomalies, dis- } \\
\text { ease symptoms, } \\
\text { and emergen- } \\
\text { cies }\end{array}$ \\
\hline $\begin{array}{l}\text { Forkan et } \\
\text { al, } 2017 \\
{[29]}\end{array}$ & $\begin{array}{l}\text { Beth Israel } \\
\text { Deaconess } \\
\text { Medical } \\
\text { Center ICU }\end{array}$ & $\begin{array}{l}\text { ICU bedside } \\
\text { monitors and } \\
\text { medical } \\
\text { records (MIM- } \\
\text { IC-II) }\end{array}$ & 4893 patients & Not specified & $\begin{array}{l}\text { Build a prognos- } \\
\text { tic model, ViSi- } \\
\text { BiD, that can ac- } \\
\text { curately identify } \\
\text { dangerous clini- } \\
\text { cal events of a } \\
\text { home-monitored } \\
\text { patient in ad- } \\
\text { vance }\end{array}$ & $\begin{array}{l}\mathrm{HR}, \mathrm{SBP}, \\
\mathrm{DBP}, \text { mean } \\
\mathrm{BP}, \mathrm{RR}, \mathrm{SpO}_{2}\end{array}$ & $\begin{array}{l}\text { Per-minute } \\
\text { sampling }\end{array}$ & $\begin{array}{l}\text { Dangerous clini- } \\
\text { cal events }\end{array}$ \\
\hline $\begin{array}{l}\text { Guillame- } \\
\text { Bert et al, } \\
2017 \text { [43] }\end{array}$ & $\begin{array}{l}\text { Step-down } \\
\text { unit }\end{array}$ & $\begin{array}{l}\text { Bedside moni- } \\
\text { tor measure- } \\
\text { ments over } 8 \\
\text { weeks }\end{array}$ & $\begin{array}{l}297 \text { admis- } \\
\text { sions }\end{array}$ & $\begin{array}{l}127 \text { patients } \\
(43 \%) \text { exhibited } \\
\text { at least } 1 \text { real } \\
\text { event during } \\
\text { their stay }\end{array}$ & $\begin{array}{l}\text { Forecast CRI uti- } \\
\text { lizing data from } \\
\text { continuous moni- } \\
\text { toring of physio- } \\
\text { logic vital sign } \\
\text { measurements }\end{array}$ & $\begin{array}{l}\mathrm{HR}, \mathrm{RR}, \\
\mathrm{SPO}_{2}, \mathrm{SBP}, \\
\text { DBP, mean } \\
\mathrm{BP}\end{array}$ & $\begin{array}{l}\text { Every } 20 \text { sec- } \\
\text { onds }(\mathrm{HR}, \\
\left.\mathrm{RR}, \mathrm{SPO}_{2}\right) \text {, } \\
\text { every } 2 \text { hours } \\
\text { (SBP, DBP, } \\
\text { and mean } \mathrm{BP} \text { ) }\end{array}$ & $\begin{array}{l}\text { At least } 1 \text { event } \\
\text { threshold limit } \\
\text { criteria exceed- } \\
\text { ed for }>80 \% \text { of } \\
\text { last } 3 \text { minutes }\end{array}$ \\
\hline $\begin{array}{l}\text { Ho et al, } \\
2017 \text { [38] }\end{array}$ & $\begin{array}{l}\text { Beth Israel } \\
\text { Deaconess } \\
\text { Medical } \\
\text { Center ICU }\end{array}$ & $\begin{array}{l}\text { ICU bedside } \\
\text { monitors and } \\
\text { medical } \\
\text { records (MIM- } \\
\text { IC-II) }\end{array}$ & 763 patients & $\begin{array}{l}197 \text { patients } \\
(25.8 \%) \text { experi- } \\
\text { enced a cardiac } \\
\text { arrest event }\end{array}$ & $\begin{array}{l}\text { Build a cardiac } \\
\text { arrest risk predic- } \\
\text { tion model capa- } \\
\text { ble of early notifi- } \\
\text { cation at time } \mathrm{z} \text { ( } \mathrm{z} \\
\geq 5 \text { hours prior to } \\
\text { the event) }\end{array}$ & $\begin{array}{l}\text { Temperature, } \\
\mathrm{SpO}_{2}, \mathrm{HR}, \\
\mathrm{RR}, \mathrm{DBP}, \\
\mathrm{SBP}, \text { pulse } \\
\text { pressure index }\end{array}$ & $\begin{array}{l}1 \text { reading per } \\
\text { hour }\end{array}$ & Cardiac arrest \\
\hline $\begin{array}{l}\text { Jang et al, } \\
2019 \text { [35] }\end{array}$ & $\begin{array}{l}\text { ED visits to } \\
\text { a tertiary } \\
\text { academic } \\
\text { hospital }\end{array}$ & $\begin{array}{l}\text { EHR data } \\
\text { from ED visits }\end{array}$ & $\begin{array}{l}\text { Nontraumatic } \\
\text { ED visits }\end{array}$ & $\begin{array}{l}374,605 \text { eligible } \\
\text { ED visits of } \\
233,763 \text { pa- } \\
\text { tients; } 1097 \\
(0.3 \%) \text { patients } \\
\text { with cardiac ar- } \\
\text { rest }\end{array}$ & $\begin{array}{l}\text { Develop and test } \\
\text { artificial neural } \\
\text { network classi- } \\
\text { fiers for early de- } \\
\text { tection of patients } \\
\text { at risk of cardiac } \\
\text { arrest in EDs }\end{array}$ & $\begin{array}{l}\text { Age, sex, } \\
\text { chief com- } \\
\text { plaint, SBP, } \\
\text { DBP, HR, RR, } \\
\text { temperature, } \\
\text { AVPU }\end{array}$ & Not specified & $\begin{array}{l}\text { Development of } \\
\text { cardiac arrest } \\
\text { within } 24 \text { hours } \\
\text { after prediction }\end{array}$ \\
\hline
\end{tabular}




\begin{tabular}{|c|c|c|c|c|c|c|c|c|}
\hline $\begin{array}{l}\text { Authors, } \\
\text { year }\end{array}$ & Setting(s) & $\begin{array}{l}\text { Data collec- } \\
\text { tion }\end{array}$ & $\begin{array}{l}\text { Cohort descrip- } \\
\text { tion }\end{array}$ & Event rate & Study purpose & Predictors & $\begin{array}{l}\text { Measurement } \\
\text { frequency }\end{array}$ & Outcome \\
\hline $\begin{array}{l}\text { Kwon et al, } \\
2018 \text { [26] }\end{array}$ & $\begin{array}{l}\text { Cardiovascu- } \\
\text { lar teaching } \\
\text { hospital and } \\
\text { community } \\
\text { general hos- } \\
\text { pital }\end{array}$ & $\begin{array}{l}\text { Data collected } \\
\text { manually by } \\
\text { staff on gener- } \\
\text { al wards, by } \\
\text { bedside moni- } \\
\text { tors in ICUs }\end{array}$ & $\begin{array}{l}52,131 \text { pa- } \\
\text { tients }\end{array}$ & $\begin{array}{l}419 \text { patients } \\
(0.8 \%) \text { with car- } \\
\text { diac arrest; } 814 \\
(1.56 \%) \text { deaths } \\
\text { without attempt- } \\
\text { ed resuscitation }\end{array}$ & $\begin{array}{l}\text { Predict whether } \\
\text { an input vector } \\
\text { belonged within } \\
\text { the prediction } \\
\text { time window } \\
\text { ( } 0.5-24 \text { hours be- } \\
\text { fore the outcome) }\end{array}$ & $\begin{array}{l}\text { SBP, HR, RR, } \\
\text { temperature }\end{array}$ & $\begin{array}{l}3 \text { times a day } \\
\text { on general } \\
\text { wards, every } \\
10 \text { minutes in } \\
\text { ICUs }\end{array}$ & $\begin{array}{l}\text { Primary out- } \\
\text { come: first car- } \\
\text { diac arrest; sec- } \\
\text { ondary out- } \\
\text { come: death } \\
\text { without attempt- } \\
\text { ed resuscitation }\end{array}$ \\
\hline $\begin{array}{l}\text { Kwon et al, } \\
2018 \text { [11] }\end{array}$ & $\begin{array}{l}151 \text { EDs in } \\
\text { Korea }\end{array}$ & $\begin{array}{l}\text { Korean Nation- } \\
\text { al Emergency } \\
\text { Department } \\
\text { Information } \\
\text { System } \\
\text { (NEDIS) }\end{array}$ & $\begin{array}{l}10,967,518 \\
\text { ED visits }\end{array}$ & $\begin{array}{l}153,217(1.4 \%) \\
\text { in-hospital } \\
\text { deaths; } 625,117 \\
(5.7 \%) \text { critical } \\
\text { care admis- } \\
\text { sions; } \\
2,964,367 \\
(27.0 \%) \text { hospi- } \\
\text { talizations }\end{array}$ & $\begin{array}{l}\text { Validate that a } \\
\text { DTAS }{ }^{n} \text { identifies } \\
\text { high-risk patients } \\
\text { more accurately } \\
\text { than existing } \\
\text { triage and acuity } \\
\text { scores }\end{array}$ & $\begin{array}{l}\text { Age, sex, } \\
\text { chief com- } \\
\text { plaint, time } \\
\text { from symptom } \\
\text { onset to ED } \\
\text { visit, arrival } \\
\text { mode, trauma, } \\
\text { initial vital } \\
\text { signs (SBP, } \\
\text { DBP, HR, RR, } \\
\text { temperature), } \\
\text { mental status }\end{array}$ & $\begin{array}{l}\text { At ED admis- } \\
\text { sion }\end{array}$ & $\begin{array}{l}\text { Primary out- } \\
\text { come: in-hospi- } \\
\text { tal mortality; } \\
\text { secondary out- } \\
\text { come: critical } \\
\text { care; tertiary } \\
\text { outcome: hospi- } \\
\text { talization }\end{array}$ \\
\hline $\begin{array}{l}\text { Larburu et } \\
\text { al, } 2018 \\
{[22]}\end{array}$ & $\begin{array}{l}\text { OSI Bilbao- } \\
\text { Basurto (Os- } \\
\text { akidetza) } \\
\text { Hospital and } \\
\text { ED admis- } \\
\text { sions, ambu- } \\
\text { latory }\end{array}$ & $\begin{array}{l}\text { Collected } \\
\text { manually by } \\
\text { clinicians and } \\
\text { patients }\end{array}$ & 242 patients & $\begin{array}{l}202 \text { predictable } \\
\text { decompensa- } \\
\text { tions }\end{array}$ & $\begin{array}{l}\text { Prevent mobile } \\
\text { heart failure pa- } \\
\text { tients' decompen- } \\
\text { sation using pre- } \\
\text { dictive models }\end{array}$ & $\begin{array}{l}\text { SBP, DBP, } \\
\mathrm{HR}, \mathrm{SaO}_{2}, \\
\text { weight }\end{array}$ & $\begin{array}{l}\text { At diagnosis } \\
\text { and 3-7 times } \\
\text { per week in } \\
\text { ambulatory } \\
\text { patients }\end{array}$ & $\begin{array}{l}\text { Heart failure } \\
\text { decompensation }\end{array}$ \\
\hline $\begin{array}{l}\text { Li et al, } \\
2016 \text { [39] }\end{array}$ & $\begin{array}{l}\text { Beth Israel } \\
\text { Deaconess } \\
\text { Medical } \\
\text { Center ICU }\end{array}$ & $\begin{array}{l}\text { ICU bedside } \\
\text { monitors and } \\
\text { medical } \\
\text { records (MIM- } \\
\text { IC-II) }\end{array}$ & 12 patients & Not specified & $\begin{array}{l}\text { Adaptive online } \\
\text { monitoring of pa- } \\
\text { tients in ICUs }\end{array}$ & $\begin{array}{l}\text { HR, SBP, } \\
\text { DBP, MAP } \\
\text { RR }\end{array}$ & $\begin{array}{l}\text { At least } 1 \\
\text { measurement } \\
\text { per hour }\end{array}$ & $\begin{array}{l}\text { Signs of deterio- } \\
\text { ration }\end{array}$ \\
\hline $\begin{array}{l}\text { Liu et al, } \\
2014 \text { [36] }\end{array}$ & $\begin{array}{l}\text { ED of a ter- } \\
\text { tiary hospital } \\
\text { in Singapore }\end{array}$ & $\begin{array}{l}\text { Manual vital } \\
\text { measurements } \\
\text { by nurses or } \\
\text { physicians }\end{array}$ & $\begin{array}{l}702 \text { patients } \\
\text { with undiffer- } \\
\text { entiated, non- } \\
\text { traumatic } \\
\text { chest pain }\end{array}$ & $\begin{array}{l}29(4.13 \%) \text { pa- } \\
\text { tients met prima- } \\
\text { ry outcome }\end{array}$ & $\begin{array}{l}\text { Discover the } \\
\text { most relevant } \\
\text { variables for risk } \\
\text { prediction of ma- } \\
\text { jor adverse car- } \\
\text { diac events using } \\
\text { clinical signs and } \\
\text { HR variability }\end{array}$ & SBP, RR, HR & Not specified & $\begin{array}{l}\text { Composite of } \\
\text { events such as } \\
\text { death and car- } \\
\text { diac arrest with- } \\
\text { in } 72 \text { hours of } \\
\text { arrival at the } \\
\text { ED }\end{array}$ \\
\hline $\begin{array}{l}\text { Mao et al, } \\
2018 \text { [34] }\end{array}$ & $\begin{array}{l}\text { ICU, inpa- } \\
\text { tient wards, } \\
\text { outpatient } \\
\text { visits }\end{array}$ & $\begin{array}{l}\text { UCSF}^{\mathrm{p}} \\
\text { dataset:inpa- } \\
\text { tient and outpa- } \\
\text { tient visits; } \\
\text { MIMIC-III: } \\
\text { ICU bedside } \\
\text { monitors }\end{array}$ & $\begin{array}{l}\text { UCSF: } 90,353 \\
\text { patients; } \\
\text { MIMIC-III: } \\
\text { 21,604 pa- } \\
\text { tients }\end{array}$ & $\begin{array}{l}\text { UCSF: } 1179 \\
(1.3 \%) \text { sepsis, } \\
349(0.39 \%) \text { se- } \\
\text { vere sepsis, } 614 \\
(0.68 \%) \text { septic } \\
\text { shock; MIMIC- } \\
\text { III: sepsis } \\
(1.91 \%), \text { severe } \\
\text { sepsis }(2.82 \%) \text {, } \\
\text { septic shock } \\
(4.36 \%)\end{array}$ & Sepsis prediction & $\begin{array}{l}\mathrm{SBP}, \mathrm{DBP}, \\
\mathrm{HR}, \mathrm{RR}, \\
\mathrm{SpO}_{2} \text {, temper- } \\
\text { ature }\end{array}$ & Hourly & $\begin{array}{l}\text { Sepsis, severe } \\
\text { sepsis, septic } \\
\text { shock }\end{array}$ \\
\hline $\begin{array}{l}\text { Olsen et al, } \\
2018 \text { [46] }\end{array}$ & $\begin{array}{l}\text { PACU }{ }^{\mathrm{q}}, \\
\text { Rigshospi- } \\
\text { talet, Univer- } \\
\text { sity of } \\
\text { Copenhagen, } \\
\text { Denmark }\end{array}$ & $\begin{array}{l}\text { IntelliVue } \\
\text { MP5, BM- } \\
\text { EYE Nexfin } \\
\text { bedside moni- } \\
\text { tors during ad- } \\
\text { mission to } \\
\text { post anesthetic } \\
\text { care unit }\end{array}$ & 178 patients & $\begin{array}{l}160(89.9 \%) \\
\text { had } \geq 1 \text { mi- } \\
\text { croevent occur- } \\
\text { ring during ad- } \\
\text { mission; } 116 \\
\text { patients } \\
(65.2 \%) \text { had } \geq 1 \\
\text { microevent with } \\
\text { a duration }>15 \\
\text { minutes }\end{array}$ & $\begin{array}{l}\text { Develop a predic- } \\
\text { tive algorithm de- } \\
\text { tecting early } \\
\text { signs of deteriora- } \\
\text { tion in the PACU } \\
\text { using continuous- } \\
\text { ly collected car- } \\
\text { diopulmonary vi- } \\
\text { tal signs }\end{array}$ & $\begin{array}{l}\mathrm{SpO}_{2}, \mathrm{SBP} \\
\mathrm{HR}, \mathrm{MAP}\end{array}$ & $\begin{array}{l}\text { Every minute } \\
\left(\mathrm{SpO}_{2}, \mathrm{SBP},\right. \\
\mathrm{HR}) \text {, every } 15 \\
\text { minutes } \\
\text { (MAP) }\end{array}$ & $\begin{array}{l}\text { Signs of deterio- } \\
\text { ration }\end{array}$ \\
\hline
\end{tabular}




\begin{tabular}{|c|c|c|c|c|c|c|c|c|}
\hline $\begin{array}{l}\text { Authors, } \\
\text { year }\end{array}$ & Setting(s) & $\begin{array}{l}\text { Data collec- } \\
\text { tion }\end{array}$ & $\begin{array}{l}\text { Cohort descrip- } \\
\text { tion }\end{array}$ & Event rate & Study purpose & Predictors & $\begin{array}{l}\text { Measurement } \\
\text { frequency }\end{array}$ & Outcome \\
\hline $\begin{array}{l}\text { Shashiku- } \\
\text { mar et al, } \\
2017 \text { [40] }\end{array}$ & $\begin{array}{l}\text { Adult ICU } \\
\text { units }\end{array}$ & $\begin{array}{l}\text { ICU bedside } \\
\text { monitors, Bed- } \\
\text { master sys- } \\
\text { tem; up to } 24 \\
\text { hours of moni- } \\
\text { toring }\end{array}$ & $\begin{array}{l}\text { Patients with } \\
\text { unselected } \\
\text { mixed surgical } \\
\text { procedures }\end{array}$ & 242 sepsis cases & $\begin{array}{l}\text { Predict onset of } \\
\text { sepsis } 4 \text { hours } \\
\text { ahead of time, us- } \\
\text { ing commonly } \\
\text { measured vital } \\
\text { signs }\end{array}$ & $\begin{array}{l}\text { MAP, HR, } \\
\mathrm{SpO}_{2}, \mathrm{SBP}, \\
\mathrm{DBP}, \mathrm{RR}, \\
\mathrm{GCS}, \text { tempera- } \\
\text { ture, comorbid- } \\
\text { ity, clinical } \\
\text { context, admis- } \\
\text { sion unit, sur- } \\
\text { gical special- } \\
\text { ty, wound } \\
\text { type, age, gen- } \\
\text { der, weight, } \\
\text { race }\end{array}$ & $\begin{array}{l}\geq 1 \text { measure- } \\
\text { ment per hour }\end{array}$ & Onset of sepsis \\
\hline $\begin{array}{l}\text { Tarassenko } \\
\text { et al, } 2006 \\
{[32]}\end{array}$ & $\begin{array}{l}\text { General } \\
\text { wards at } \\
\text { John Rad- } \\
\text { cliffe Hospi- } \\
\text { tal in Ox- } \\
\text { ford, United } \\
\text { Kingdom }\end{array}$ & $\begin{array}{l}\text { Bedside moni- } \\
\text { tors for at } \\
\text { least } 24 \text { hours } \\
\text { per patient }\end{array}$ & $\begin{array}{l}150 \text { general- } \\
\text { ward patients }\end{array}$ & Not specified & $\begin{array}{l}\text { A real-time auto- } \\
\text { mated system, } \\
\text { BioSign, which } \\
\text { tracks patient sta- } \\
\text { tus by combining } \\
\text { information from } \\
\text { vital signs }\end{array}$ & $\begin{array}{l}\mathrm{HR}, \mathrm{RR}, \\
\mathrm{SpO}_{2} \text {, skin } \\
\text { temperature, } \\
\text { average SBP - } \\
\text { average DBP }\end{array}$ & $\begin{array}{l}\text { Every } 30 \text { min- } \\
\text { utes }(\mathrm{BP}), \text { ev- } \\
\text { ery } 5 \text { seconds } \\
\text { (other vitals) }\end{array}$ & $\begin{array}{l}\text { Signs of deterio- } \\
\text { ration }\end{array}$ \\
\hline $\begin{array}{l}\text { Van Wyk } \\
\text { et al, } 2017 \\
{[33]}\end{array}$ & $\begin{array}{l}\text { Methodist } \\
\text { LeBonheur } \\
\text { Hospital, } \\
\text { Memphis, } \\
\text { TN }\end{array}$ & $\begin{array}{l}\text { Bedside moni- } \\
\text { tors: Cerner } \\
\text { CareAware } \\
\text { iBus system }\end{array}$ & 2995 patients & $\begin{array}{l}343 \text { patients } \\
(11.5 \%) \text { diag- } \\
\text { nosed with sep- } \\
\text { sis }\end{array}$ & $\begin{array}{l}\text { Classify patients } \\
\text { into sepsis and } \\
\text { nonsepsis groups } \\
\text { using data collect- } \\
\text { ed at various fre- } \\
\text { quencies from the } \\
\text { first } 12 \text { hours af- } \\
\text { ter admission }\end{array}$ & $\begin{array}{l}\mathrm{HR}, \mathrm{MAP}, \\
\mathrm{DBP}, \mathrm{SBP}, \\
\mathrm{SpO}_{2} \text {, age, } \\
\text { race, gender, } \\
\text { fraction of in- } \\
\text { spired oxygen }\end{array}$ & Every minute & Sepsis detection \\
\hline $\begin{array}{l}\text { Yoon et al, } \\
2019[41]\end{array}$ & $\begin{array}{l}\text { Beth Israel } \\
\text { Deaconess } \\
\text { Medical } \\
\text { Center ICU }\end{array}$ & $\begin{array}{l}\text { ICU bedside } \\
\text { monitors and } \\
\text { medical } \\
\text { records (MIM- } \\
\text { IC-II) }\end{array}$ & 2809 subjects & $\begin{array}{l}787 \text { tachycardia } \\
\text { episodes }\end{array}$ & $\begin{array}{l}\text { Predicting tachy- } \\
\text { cardia as a surro- } \\
\text { gate for instabili- } \\
\text { ty }\end{array}$ & $\begin{array}{l}\text { Arterial DBP, } \\
\text { arterial SBP, } \\
\mathrm{HR}, \mathrm{RR}, \\
\mathrm{SpO}_{2}, \mathrm{MAP}\end{array}$ & $\begin{array}{l}1 / 60 \mathrm{~Hz} \text { or } 1 \\
\mathrm{~Hz}\end{array}$ & $\begin{array}{l}\text { Tachycardia } \\
\text { episode }\end{array}$ \\
\hline
\end{tabular}

${ }^{\mathrm{a}} \mathrm{ICU}$ : intensive care unit.

${ }^{b}$ NEWS: National Early Warning Score.

${ }^{\mathrm{c}} \mathrm{HR}$ : heart rate.

${ }^{d} \mathrm{RR}$ : respiratory rate.

${ }^{\mathrm{e}} \mathrm{SBP}$ : systolic blood pressure.

${ }^{\mathrm{f}}$ AVPU: alert, verbal, pain, unresponsive.

${ }^{\mathrm{g}} \mathrm{CRI}$ : cardiorespiratory instability.

${ }^{\mathrm{h}}$ DBP: diastolic blood pressure.

${ }^{\mathrm{i}} \mathrm{ED}$ : emergency department.

${ }^{\mathrm{j}} \mathrm{EHR}$ : electronic health record.

${ }^{\mathrm{k}}$ GCS: Glasgow Coma Score.

${ }^{1}$ NHS: National Health Service.

m MIMIC: Medical Information Mart for Intensive Care.

${ }^{\mathrm{n}}$ DTAS: Deep learning-based Triage and Acuity Score.

${ }^{\circ}$ MAP: mean arterial pressure.

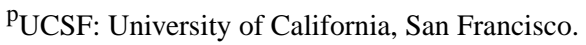

${ }^{\mathrm{q}} \mathrm{PACU}$ : postanesthesia care unit.

\section{Predictor Variables}

The most commonly used vital sign predictors were HR, RR, systolic $\mathrm{BP}$, diastolic $\mathrm{BP}, \mathrm{SpO}_{2}$, body temperature, level of consciousness through either the Glasgow Coma Score or the AVPU scale, and mean arterial pressure. Measurement frequencies for these variables ranged from once every 5 seconds
[32] in hospital wards to 3-7 times per week [22] in an ambulatory setting. Other commonly used predictors included age, gender, weight, ethnicity, chief complaint, and comorbidities. 


\section{Outcomes}

The outcomes being predicted in most studies focused on cardiorespiratory insufficiency-related events. Cardiac arrest was the primary outcome in $7[24,26,35,36,38,42,45]$ studies, while general cardiorespiratory deterioration or decompensation was the primary outcome in 5 studies $[25,39,41,43,44]$. Another commonly predicted outcome was sepsis, which included the time of onset of sepsis [34,37,40], severe sepsis [33,34], septic shock [34], and sepsis-related mortality [23]. Other outcomes explored within the studies include unanticipated ICU admissions [24,42,45], development of critical illness [24], general physiological deterioration $[25,32,39,46]$, abnormal or dangerous clinical events [27-29], and mortality [11,24,42].

Outcomes were first identified, and baseline models were created using predefined parameter thresholds (ground truth) consistent with the MEWS [23,26,35] or NEWS [23,42,46] criteria for cardiorespiratory instability and general physiological deterioration, while the sepsis-related outcomes were identified based on the thresholds set within the systemic inflammatory response syndrome [34], quick Sequential Organ Failure Assessment (qSOFA) [23], and SOFA [37] criteria. Some studies [22,27-29,43,44] also used thresholds and criteria based on the population served by their individual care setting.

\section{Models and Performance}

All included studies consider the prediction of deterioration risk to be a classification task and therefore use different types of classification models in the process, including tree-based models, linear models, kernel-based methods, and neural networks (refer to Table 2 for a full inventory of methods used, model performance achieved, and prediction windows, and see Multimedia Appendix 2 for a description of ML methods).

Measures used to assess model performance varied across the studies. The most common measure was the area under the receiver operator characteristic (AUROC) along with model accuracy, sensitivity, and specificity. Area under the precision-recall, F-score, Hamming's score, and precision (positive predictive value) were reported less commonly.

Prediction windows ranged from 30 minutes to 30 days before an event.

Model performance varied substantially based on outcome measure being predicted (eg, cardiorespiratory insufficiency vs sepsis), ML method used (eg, linear vs tree-based), and prediction window (eg, 30 minutes before an event vs 4 hours before). 
Table 2. Machine learning (ML) models and comparisons used for outcome prediction.

\begin{tabular}{|c|c|c|c|c|c|c|c|c|}
\hline Study & Cohort & Event rate & ML model(s) & $\begin{array}{l}\text { Missing data } \\
\text { handling }\end{array}$ & $\begin{array}{l}\text { Best ML model } \\
\text { performance }\end{array}$ & $\begin{array}{l}\text { ML model com- } \\
\text { parisons }\end{array}$ & $\begin{array}{l}\text { Predic- } \\
\text { tion win- } \\
\text { dow }\end{array}$ & $\begin{array}{l}\text { Aggregate } \\
\text { weighted } \\
\text { EWS }{ }^{\text {a compar- }} \\
\text { isons }\end{array}$ \\
\hline $\begin{array}{l}\text { Badriyah et } \\
\text { al, } 2014 \text { [45] }\end{array}$ & $\begin{array}{l}35,585 \text { ad- } \\
\text { missions }\end{array}$ & $\begin{array}{l}199(0.56 \%) \text {, car- } \\
\text { diac arrest; } \\
1161(3.26 \%) \\
\text { unanticipated } \\
\text { ICU }^{\text {b }} \text { admissions; } \\
1789(5.02 \%) \\
\text { deaths; } 3149 \\
(8.85 \%) \text { any out- } \\
\text { come }\end{array}$ & $\begin{array}{l}\text { Decision tree } \\
\text { analysis }\end{array}$ & $\begin{array}{l}\text { Not speci- } \\
\text { fied }\end{array}$ & $\begin{array}{l}\text { Decision tree pre- } \\
\text { dicted cardiac ar- } \\
\text { rest: AU- } \\
\text { ROC }^{\mathrm{C}}=0.708 ; \\
\text { unanticipated ICU } \\
\text { admission: AU- } \\
\text { ROC=0.862; } \\
\text { death: AU- } \\
\text { ROC }=0.899 ; \text { any } \\
\text { outcomes: AU- } \\
\text { ROC }=0.877\end{array}$ & Not specified & $\begin{array}{l}\text { Within } 24 \\
\text { hours pre- } \\
\text { ceding } \\
\text { events }\end{array}$ & $\begin{array}{l}\text { NEWS }{ }^{\mathrm{d}} \text { AU- } \\
\text { ROC: cardiac } \\
\text { arrest, } 0.722 ; \\
\text { unanticipated } \\
\text { ICU admis- } \\
\text { sion, } 0.857 ; \\
\text { death, } 0.894 ; \\
\text { any outcomes, } \\
0.873\end{array}$ \\
\hline $\begin{array}{l}\text { Chen at al, } \\
2017 \text { [44] }\end{array}$ & $\begin{array}{l}1880 \text { pa- } \\
\text { tients ( } 1971 \\
\text { admissions) }\end{array}$ & $\begin{array}{l}997 \text { patients } \\
(53 \%) \text { or } 1056 \\
\text { admissions } \\
(53.6 \%) \text { who ex- } \\
\text { perienced CRI } \\
\text { events }\end{array}$ & $\begin{array}{l}\text { Variant of the } \\
\text { random forest } \\
\text { classification } \\
\text { model using } \\
\text { nonrandom } \\
\text { splits }\end{array}$ & $\begin{array}{l}\text { Not speci- } \\
\text { fied }\end{array}$ & $\begin{array}{l}\text { Random forest } \\
\text { AUC }{ }^{f} \text { initially re- } \\
\text { mained constant } \\
(0.58-0.60), \text { fol- } \\
\text { lowed by an in- } \\
\text { creasing trend, } \\
\text { with AUCs rising } \\
\text { from } 0.57 \text { to } 0.89 \\
\text { during the } 4 \text { hours } \\
\text { immediately pre- } \\
\text { ceding events }\end{array}$ & $\begin{array}{l}\text { Logistic regres- } \\
\text { sion: } A U C=0.7 \\
\text { lasso logistic re- } \\
\text { gression: } \\
\text { AUC }=0.82\end{array}$ & $\begin{array}{l}\text { Within } 4 \\
\text { hours pre- } \\
\text { ceding } \\
\text { events }\end{array}$ & $\begin{array}{l}\text { No compari- } \\
\text { son }\end{array}$ \\
\hline $\begin{array}{l}\text { Churpek et } \\
\text { al, } 2016 \text { [24] }\end{array}$ & $\begin{array}{l}269,999 \text { ad- } \\
\text { missions }\end{array}$ & $\begin{array}{l}16,452 \text { outcomes } \\
(6.09 \%)\end{array}$ & $\begin{array}{l}\text { Univariate } \\
\text { analysis, bi- } \\
\text { variate analy- } \\
\text { sis }\end{array}$ & $\begin{array}{l}\text { Forward im- } \\
\text { putation, me- } \\
\text { dian value } \\
\text { imputation }\end{array}$ & $\begin{array}{l}\text { Trends increased } \\
\text { model accuracy } \\
\text { compared to a } \\
\text { model containing } \\
\text { only current vital } \\
\text { signs (AUC } 0.78 \\
\text { vs } 0.74 \text { ); vital sign } \\
\text { slope improved } \\
\text { AUC by } 0.013\end{array}$ & Not specified & $\begin{array}{l}\text { Within } 4 \\
\text { hours pre- } \\
\text { ceding } \\
\text { events }\end{array}$ & $\begin{array}{l}\text { No compari- } \\
\text { son }\end{array}$ \\
\hline $\begin{array}{l}\text { Chiew et al, } \\
2019 \text { [23] }\end{array}$ & 214 patients & $\begin{array}{l}40 \text { patients } \\
(18.7 \%) \text { met out- } \\
\text { come }\end{array}$ & $\begin{array}{l}\text { K-nearest } \\
\text { neighbor, ran- } \\
\text { dom forest, } \\
\text { adaptive } \\
\text { boosting, gra- } \\
\text { dient boost- } \\
\text { ing, support } \\
\text { vector ma- } \\
\text { chine }\end{array}$ & $\begin{array}{l}\text { Not speci- } \\
\text { fied }\end{array}$ & 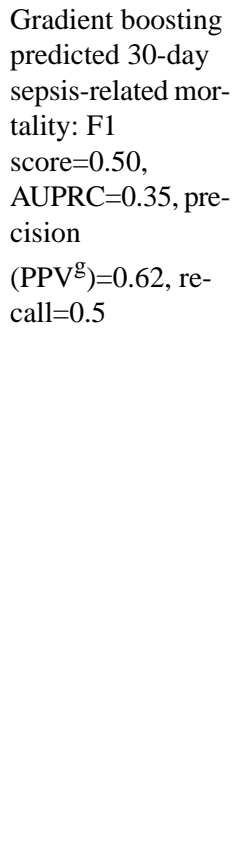 & $\begin{array}{l}\text { K-nearest neigh- } \\
\text { bor: F1 } \\
\text { score }=0.10, \\
\text { AUPRC }=0.10, \\
\text { precision } \\
\text { (PPV)=0.33, re- } \\
\text { call=0.6; random } \\
\text { forest: F1 } \\
\text { score }=0.35, \\
\text { AUPRC }=0.27, \\
\text { precision } \\
\text { (PPV)=0.26, re- } \\
\text { call=0.56; adap- } \\
\text { tive boosting: F1 } \\
\text { score }=0.40, \\
\text { AUPRC }=0.31, \\
\text { precision } \\
(P P V)=0.43, \text { re- } \\
\text { call=0.38; SVM }{ }^{\mathrm{h}} \text { : } \\
\text { F1 score }=0.43, \\
\text { AUPRC }=0.29, \\
\text { precision } \\
(P P V)=0.33, \text { re- } \\
\text { call=0.63 }\end{array}$ & $\begin{array}{l}\text { Within } 30 \\
\text { days pre- } \\
\text { ceding } \\
\text { event }\end{array}$ & $\begin{array}{l}\text { SEDS }: \\
\text { F1=0.40, } \\
\text { AUPRC=0.22; } \\
\text { qSOFA }^{j} \text { : } \\
\text { F1=0.32, } \\
\text { AUPRC=0.21; } \\
\text { NEWS; } \\
\text { F1=0.38, } \\
\text { AUPRC }=0.28 ; \\
\text { MEWS }: \\
\text { F1=0.30, } \\
\text { AUPRC }=0.25\end{array}$ \\
\hline
\end{tabular}




\begin{tabular}{|c|c|c|c|c|c|c|c|c|}
\hline Study & Cohort & Event rate & ML model(s) & $\begin{array}{l}\text { Missing data } \\
\text { handling }\end{array}$ & $\begin{array}{l}\text { Best ML model } \\
\text { performance }\end{array}$ & $\begin{array}{l}\text { ML model com- } \\
\text { parisons }\end{array}$ & $\begin{array}{l}\text { Predic- } \\
\text { tion win- } \\
\text { dow }\end{array}$ & $\begin{array}{l}\text { Aggregate } \\
\text { weighted } \\
\text { EWS }^{\text {a compar- }} \\
\text { isons }\end{array}$ \\
\hline $\begin{array}{l}\text { Chiu et al, } \\
2019 \text { [42] }\end{array}$ & $\begin{array}{l}\text { Adults under- } \\
\text { going risk- } \\
\text { stratified ma- } \\
\text { jor cardiac } \\
\text { surgery } \\
(\mathrm{n}=13,631)\end{array}$ & $\begin{array}{l}578 \text { patients } \\
(4.2 \%) \text { with an } \\
\text { outcome; } 499 \text { pa- } \\
\text { tients }(3.66 \%) \\
\text { with unplanned } \\
\text { ICU readmissions }\end{array}$ & $\begin{array}{l}\text { Logistic re- } \\
\text { gression }\end{array}$ & $\begin{array}{l}\text { Observations } \\
\text { with missing } \\
\text { values were } \\
\text { excluded }\end{array}$ & $\begin{array}{l}\text { Logistic regression } \\
\text { predicted the event } \\
24 \text { hours in ad- } \\
\text { vance: } \mathrm{AU}- \\
\text { ROC }=0.779 ; 12 \\
\text { hours in advance: } \\
\text { AUROC }=0.815 ; 6 \\
\text { hours in advance: } \\
\text { AUROC }=0.841\end{array}$ & Not specified & $\begin{array}{l}\text { Within } \\
24,12, \\
\text { and } 6 \\
\text { hours pre- } \\
\text { ceding } \\
\text { event }\end{array}$ & $\begin{array}{l}\text { NEWS: } 24 \\
\text { hours before } \\
\text { event, } \\
\text { AU- } \\
\text { ROC=0.754; } \\
12 \text { hours be- } \\
\text { fore event, } \\
\text { AU- } \\
\text { ROC=0.789; } 6 \\
\text { hours before } \\
\text { event, AU- } \\
\text { ROC }=0.813\end{array}$ \\
\hline $\begin{array}{l}\text { Clifton et al, } \\
2014 \text { [25] }\end{array}$ & $\begin{array}{l}200 \text { patients } \\
\text { in the postop- } \\
\text { erative ward } \\
\text { following } \\
\text { upper gas- } \\
\text { trointestinal } \\
\text { cancer } \\
\text { surgery }\end{array}$ & Not specified & $\begin{array}{l}\text { Classifiers, } \\
\text { Gaussian pro- } \\
\text { cess, one-class } \\
\text { support vector } \\
\text { machine, ker- } \\
\text { nel estimate }\end{array}$ & $\begin{array}{l}\text { Missing } \\
\text { channels re- } \\
\text { placed by } \\
\text { mean of that } \\
\text { channel }\end{array}$ & $\begin{array}{l}\text { SVM predicted de- } \\
\text { terioration: accura- } \\
\text { cy }=0.94, \text { partial } \\
\text { AUC }=0.28, \text { sensi- } \\
\text { tivity }=0.96, \text { speci- } \\
\text { ficity }=0.93\end{array}$ & $\begin{array}{l}\text { Conventional } \\
\text { SVM: accura- } \\
\text { cy }=0.90, \text { partial } \\
\text { AUC }=0.26 \text {, sensi- } \\
\text { tivity }=0.92, \\
\text { specificity }=0.87 \text {; } \\
\text { Gaussian mixture } \\
\text { models: accura- } \\
\text { cy }=0.9, \text { partial } \\
\text { AUC }=0.24, \text { sensi- } \\
\text { tivity }=0.97, \\
\text { specificity }=0.84 ; \\
\text { Gaussian process- } \\
\text { es: accura- } \\
\text { cy }=0.90, \text { partial } \\
\text { AUC }=0.26, \text { sensi- } \\
\text { tivity }=0.91, \\
\text { specificity }=0.89 ; \\
\text { kernel density es- } \\
\text { timate: accura- } \\
\text { cy }=0.91, \text { partial } \\
\text { AUC }=0.26, \text { sensi- } \\
\text { tivity }=0.94, \\
\text { specificity }=0.87\end{array}$ & $\begin{array}{l}\text { Not speci- } \\
\text { fied }\end{array}$ & $\begin{array}{l}\text { No compari- } \\
\text { son }\end{array}$ \\
\hline $\begin{array}{l}\text { Desautels et } \\
\text { al, } 2016 \text { [37] }\end{array}$ & $\begin{array}{l}22,853 \mathrm{ICU} \\
\text { stays }\end{array}$ & $\begin{array}{l}2577(11.28 \%) \\
\text { stays with con- } \\
\text { firmed sepsis }\end{array}$ & $\begin{array}{l}\text { Insight classifi- } \\
\text { er }\end{array}$ & $\begin{array}{l}\text { Carry for- } \\
\text { ward imputa- } \\
\text { tion }\end{array}$ & $\begin{array}{l}\text { Classifier predicts } \\
\text { sepsis at onset: } \\
\text { AUROC }=0.880 \text {, } \\
\mathrm{APR}^{1}=0.6 \text {, accura- } \\
\text { cy }=0.8 \text {; classifier } \\
\text { predicts sepsis } 4 \\
\text { hours before onset: } \\
\text { AUROC }=0.74 \text {, } \\
\text { APR }=0.28, \text { accura- } \\
\text { cy }=0.57\end{array}$ & Not specified & $\begin{array}{l}\text { Within } 4 \\
\text { hours pre- } \\
\text { ceding } \\
\text { event and } \\
\text { at time of } \\
\text { event on- } \\
\text { set }\end{array}$ & $\begin{array}{l}\mathrm{SIRS}^{\mathrm{m}}: \mathrm{AU}- \\
\mathrm{ROC}=0.609 \\
\text { APR=0.160; } \\
\text { qSOFA: AU- } \\
\text { ROC=0.772, } \\
\text { APR=0.277; } \\
\text { MEWS: AU- } \\
\text { ROC=0.803, } \\
\text { APR=0.327; } \\
\text { SAPS }{ }^{\mathrm{II}}: \\
\text { AU- } \\
\text { ROC=0.700, } \\
\text { APR=0.225; } \\
\text { SOFA: AU- } \\
\text { ROC=0.725, } \\
\text { APR=0.284 }\end{array}$ \\
\hline
\end{tabular}




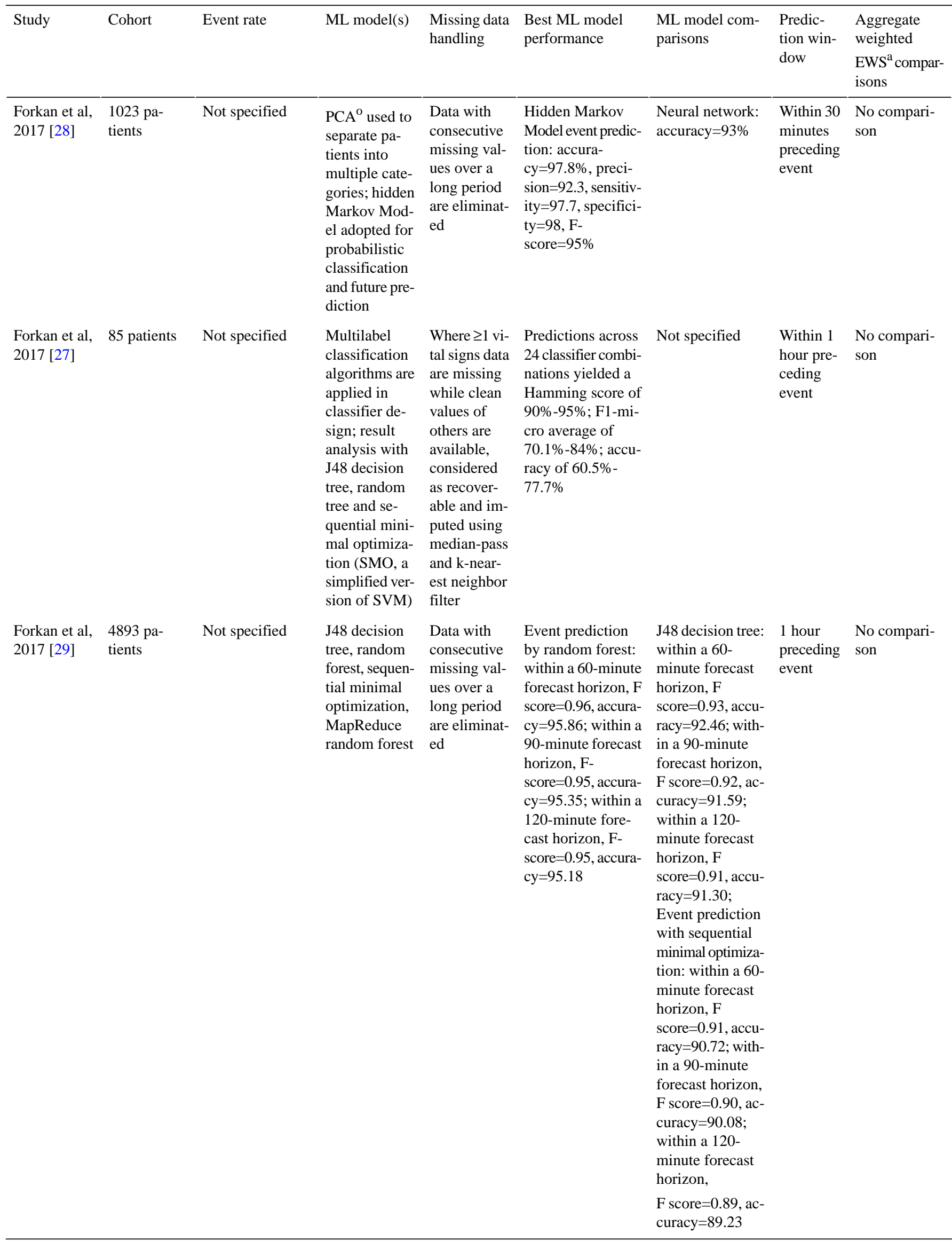




\begin{tabular}{|c|c|c|c|c|c|c|c|c|}
\hline Study & Cohort & Event rate & ML model(s) & $\begin{array}{l}\text { Missing data } \\
\text { handling }\end{array}$ & $\begin{array}{l}\text { Best ML model } \\
\text { performance }\end{array}$ & $\begin{array}{l}\text { ML model com- } \\
\text { parisons }\end{array}$ & $\begin{array}{l}\text { Predic- } \\
\text { tion win- } \\
\text { dow }\end{array}$ & $\begin{array}{l}\text { Aggregate } \\
\text { weighted } \\
\text { EWS }^{\text {a compar- }} \\
\text { isons }\end{array}$ \\
\hline $\begin{array}{l}\text { Guillame- } \\
\text { Bert et al, } \\
2017 \text { [43] }\end{array}$ & $\begin{array}{l}297 \text { admis- } \\
\text { sions }\end{array}$ & $\begin{array}{l}127 \text { patients } \\
(43 \%) \text { exhibited } \\
\text { at least } 1 \text { real } \\
\text { CRI event during } \\
\text { their stay in the } \\
\text { step-down unit }\end{array}$ & $\begin{array}{l}\text { TITA }^{\mathrm{p}} \text { rules, } \\
\text { rule fusion al- } \\
\text { gorithm; map- } \\
\text { ping function } \\
\text { from rule- } \\
\text { based features } \\
\text { to forecast } \\
\text { model learned } \\
\text { using random } \\
\text { forest classifi- } \\
\text { er }\end{array}$ & $\begin{array}{l}\text { Not speci- } \\
\text { fied }\end{array}$ & $\begin{array}{l}\text { Event forecast alert } \\
\text { within } 17 \text { minutes, } \\
51 \text { seconds before } \\
\text { onset of CRI (false } \\
\text { alert every } 12 \\
\text { hours); event fore- } \\
\text { cast alert within } 10 \\
\text { minutes, } 58 \text { sec- } \\
\text { onds before onset } \\
\text { of CRI (false alert } \\
\text { every } 24 \text { hours) }\end{array}$ & $\begin{array}{l}\text { Random forest: } \\
\text { event forecast } \\
\text { alert within } 11 \\
\text { minutes, } 25 \mathrm{sec} \text { - } \\
\text { onds before onset } \\
\text { of CRI (false } \\
\text { alert every } 12 \\
\text { hours); event } \\
\text { forecast alert } \\
\text { within } 5 \text { minutes, } \\
52 \text { seconds be- } \\
\text { fore onset of CRI } \\
\text { (false alert every } \\
24 \text { hours) }\end{array}$ & $\begin{array}{l}\text { Within } 17 \\
\text { minutes, } \\
51 \text { sec- } \\
\text { onds pre- } \\
\text { ceding } \\
\text { CRI onset }\end{array}$ & $\begin{array}{l}\text { No compari- } \\
\text { son }\end{array}$ \\
\hline $\begin{array}{l}\text { Ho et al, } \\
2017 \text { [38] }\end{array}$ & 763 patients & $\begin{array}{l}197 \text { patients } \\
(25.8 \%) \text { experi- } \\
\text { enced a cardiac } \\
\text { arrest event }\end{array}$ & $\begin{array}{l}\text { Temporal } \\
\text { transfer learn- } \\
\text { ing-based } \\
\text { model (TTL- } \\
\text { Reg) }\end{array}$ & $\begin{array}{l}\text { Imputed val- } \\
\text { ues based on } \\
\text { the median } \\
\text { from patients } \\
\text { of the same } \\
\text { gender and } \\
\text { similar ages }\end{array}$ & $\begin{array}{l}\text { TTL-Reg predicts } \\
\text { events with an } \\
\text { AUC of } 0.63\end{array}$ & Not specified & $\begin{array}{l}\text { Within } 6 \\
\text { hours pre- } \\
\text { ceding } \\
\text { event }\end{array}$ & $\begin{array}{l}\text { No compari- } \\
\text { son }\end{array}$ \\
\hline $\begin{array}{l}\text { Jang et al, } \\
2019 \text { [35] }\end{array}$ & $\begin{array}{l}\text { Non-traumat- } \\
\text { ic ED visits }\end{array}$ & $\begin{array}{l}374,605 \text { eligible } \\
\text { ED visits of } \\
233,763 \text { patients; } \\
1097(0.3 \%) \text { pa- } \\
\text { tients with car- } \\
\text { diac arrest }\end{array}$ & $\begin{array}{l}\text { ANN }^{\mathrm{q}} \text { with } \\
\text { multilayer per- } \\
\text { ceptron, ANN } \\
\text { with LSTM }{ }^{\mathrm{r}} \text {, } \\
\text { hybrid ANN; } \\
\text { comparison } \\
\text { with random } \\
\text { forest and lo- } \\
\text { gistic regres- } \\
\text { sion }\end{array}$ & $\begin{array}{l}\text { Not speci- } \\
\text { fied }\end{array}$ & $\begin{array}{l}\text { Event prediction: } \\
\text { ANN with multilay- } \\
\text { er perceptron, AU- } \\
\text { ROC }=0.929 \text {; ANN } \\
\text { with LSTM, AU- } \\
\text { ROC }=0.933 \text {; hy- } \\
\text { brid ANN, AU- } \\
\text { ROC }=0.936\end{array}$ & $\begin{array}{l}\text { Random forest, } \\
\text { AUROC }=0.923 \text {; } \\
\text { logistic regres- } \\
\text { sion, AU- } \\
\text { ROC }=0.914\end{array}$ & $\begin{array}{l}\text { Within } 24 \\
\text { hours pre- } \\
\text { ceding } \\
\text { event }\end{array}$ & $\begin{array}{l}\text { MEWS: AU- } \\
\text { ROC=0.886 }\end{array}$ \\
\hline $\begin{array}{l}\text { Kwon et al, } \\
2018 \text { [26] }\end{array}$ & $\begin{array}{l}52,131 \text { pa- } \\
\text { tients }\end{array}$ & $\begin{array}{l}419 \text { patients } \\
(0.8 \%) \text { with car- } \\
\text { diac arrest; } 814 \\
(1.56 \%) \text { deaths } \\
\text { without attempt- } \\
\text { ed resuscitation }\end{array}$ & $\begin{array}{l}3 \mathrm{RNN}^{\mathrm{S}} \text { layers } \\
\text { with LSTM to } \\
\text { deal with time } \\
\text { series data; } \\
\text { compared to } \\
\text { random forest } \\
\text { and logistic re- } \\
\text { gression }\end{array}$ & $\begin{array}{l}\text { Most recent } \\
\text { value was } \\
\text { used; if no } \\
\text { value avail- } \\
\text { able, then } \\
\text { median val- } \\
\text { ue used }\end{array}$ & $\begin{array}{l}\text { Event prediction: } \\
\text { RNNs, AU- } \\
\text { ROC }=0.85, \\
\text { AUPRC }^{\mathrm{t}}=0.044\end{array}$ & $\begin{array}{l}\text { Random forest, } \\
\text { AUROC }=0.78, \\
\text { AUPRC }=0.014 ; \\
\text { logistic regres- } \\
\text { sion, AU- } \\
\text { ROC }=0.613, \\
\text { AUPRC }=0.007\end{array}$ & $\begin{array}{l}30 \text { min- } \\
\text { utes to } 24 \\
\text { hours pre- } \\
\text { ceding } \\
\text { event }\end{array}$ & $\begin{array}{l}\text { MEWS: AU- } \\
\text { ROC }=0.603, \\
\text { AUPRC }=0.003\end{array}$ \\
\hline $\begin{array}{l}\text { Kwon et al, } \\
2018 \text { [11] }\end{array}$ & $\begin{array}{l}10,967,518 \\
\text { ED visits }\end{array}$ & $\begin{array}{l}153,217(1.4 \%) \\
\text { in-hospital } \\
\text { deaths; } 625,117 \\
(5.7 \%) \text { critical } \\
\text { care admissions; } \\
2,964,367 \\
(27.0 \%) \text { hospital- } \\
\text { izations }\end{array}$ & $\begin{array}{l}\text { DTAS }^{\mathrm{u}} \text { using } \\
\text { multilayer per- } \\
\text { ceptron with } 5 \\
\text { hidden } \\
\text { layers }\end{array}$ & Excluded & $\begin{array}{l}\text { Event prediction: } \\
\text { DTAS using multi- } \\
\text { layer perceptron, } \\
\text { AUROC }=0.935 \text {, } \\
\text { AUPRC }=0.264\end{array}$ & $\begin{array}{l}\text { Random forest: } \\
\text { AUROC }=0.89 \text {, } \\
\text { AUPRC }=0.14 ; \\
\text { logistic regres- } \\
\text { sion: AUROC= } \\
0.89, \\
\text { AUPRC }=0.16\end{array}$ & $\begin{array}{l}\text { Not speci- } \\
\text { fied }\end{array}$ & $\begin{array}{l}\text { Korean triage } \\
\text { and acuity } \\
\text { score: } \text { AU- } \\
\text { ROC }=0.785 \text {, } \\
\text { AUPRC }=0.192 \text {; } \\
\text { MEWS: AU- } \\
\text { ROC }=0.810, \\
\text { AUPRC }=0.116 ;\end{array}$ \\
\hline $\begin{array}{l}\text { Larburu et } \\
\text { al, } 2018 \text { [22] }\end{array}$ & 242 patients & $\begin{array}{l}202 \text { predictable } \\
\text { decompensations }\end{array}$ & $\begin{array}{l}\text { Naïve Bayes, } \\
\text { decision tree, } \\
\text { random forest, } \\
\text { SVM }\end{array}$ & $\begin{array}{l}\text { Not speci- } \\
\text { fied }\end{array}$ & $\begin{array}{l}\text { Decompensation } \\
\text { event prediction: } \\
\text { naïve Bayes, } \\
\text { AUC }=67 \%\end{array}$ & $\begin{array}{l}\text { Decision tree, } \\
\text { neural network, } \\
\text { random forest, } \\
\text { support vector } \\
\text { machine, stochas- } \\
\text { tic gradient de- } \\
\text { scent }\end{array}$ & $\begin{array}{l}\text { Not speci- } \\
\text { fied }\end{array}$ & $\begin{array}{l}\text { No compari- } \\
\text { son }\end{array}$ \\
\hline
\end{tabular}




\begin{tabular}{|c|c|c|c|c|c|c|c|c|}
\hline Study & Cohort & Event rate & ML model(s) & $\begin{array}{l}\text { Missing data } \\
\text { handling }\end{array}$ & $\begin{array}{l}\text { Best ML model } \\
\text { performance }\end{array}$ & $\begin{array}{l}\text { ML model com- } \\
\text { parisons }\end{array}$ & $\begin{array}{l}\text { Predic- } \\
\text { tion win- } \\
\text { dow }\end{array}$ & $\begin{array}{l}\text { Aggregate } \\
\text { weighted } \\
\text { EWS }^{\text {a compar- }} \\
\text { isons }\end{array}$ \\
\hline $\begin{array}{l}\text { Li et al, } \\
2016 \text { [39] }\end{array}$ & 12 patients & Not specified & $\begin{array}{l}\text { L-PCA (com- } \\
\text { bination of } \\
\text { just-in-time } \\
\text { learning and } \\
\text { PCA) }\end{array}$ & $\begin{array}{l}\text { Not speci- } \\
\text { fied }\end{array}$ & $\begin{array}{l}\text { Fault detection rate } \\
\text { with L-PCA: } 20 \% \\
\text { higher than with } \\
\text { PCA; } 47 \% \text { higher } \\
\text { than with fast } \\
\text { moving-window } \\
\text { PCA; best detec- } \\
\text { tion rate achieved } \\
\text { was } 99.8 \%\end{array}$ & Not specified & $\begin{array}{l}\text { Not speci- } \\
\text { fied }\end{array}$ & $\begin{array}{l}\text { No compari- } \\
\text { son }\end{array}$ \\
\hline $\begin{array}{l}\text { Liu et al, } \\
2014 \text { [36] }\end{array}$ & $\begin{array}{l}702 \text { patients } \\
\text { with undiffer- } \\
\text { entiated, } \\
\text { non-traumat- } \\
\text { ic chest pain }\end{array}$ & $\begin{array}{l}29(4.13 \%) \text { pa- } \\
\text { tients met prima- } \\
\text { ry outcome }\end{array}$ & $\begin{array}{l}\text { Novel variable } \\
\text { selection } \\
\text { framework } \\
\text { based on en- } \\
\text { semble learn- } \\
\text { ing; random } \\
\text { forest was the } \\
\text { independent } \\
\text { variable selec- } \\
\text { tor for creat- } \\
\text { ing the deci- } \\
\text { sion ensemble }\end{array}$ & $\begin{array}{l}\text { Not speci- } \\
\text { fied }\end{array}$ & $\begin{array}{l}\text { Event prediction } \\
\text { with ensemble } \\
\text { learning model: } \\
\text { AUC }=0.812 \text {, cut- } \\
\text { off score }=43 \text {, sensi- } \\
\text { tivity }=82.8 \% \text {, } \\
\text { specificity }=63.4 \%\end{array}$ & Not specified & $\begin{array}{l}\text { Within } 72 \\
\text { hours of } \\
\text { arrival at } \\
\text { ED }\end{array}$ & $\begin{array}{l}\text { TIMI }^{\mathrm{V}}: \\
\text { AUC=0.637; } \\
\text { MEWS: } \\
\text { AUC=0.622 }\end{array}$ \\
\hline $\begin{array}{l}\text { Mao et al, } \\
2018 \text { [34] }\end{array}$ & $\begin{array}{l}\mathrm{UCSF}^{\mathrm{W}}: \\
90,353 \text { pa- } \\
\text { tients; MIM- } \\
\text { IC }^{\mathrm{X}} \text {-III: } \\
21,604 \text { pa- } \\
\text { tients }\end{array}$ & $\begin{array}{l}\text { UCSF: } 1179 \\
(1.3 \%) \text { sepsis, } \\
349(0.39 \%) \text { se- } \\
\text { vere sepsis, } 614 \\
(0.68 \%) \text { septic } \\
\text { shock; MIMIC- } \\
\text { III: sepsis } \\
(1.91 \%), \text { severe } \\
\text { sepsis }(2.82 \%) \text {, } \\
\text { septic shock } \\
(4.36 \%)\end{array}$ & $\begin{array}{l}\text { Gradient tree } \\
\text { boosting + } \\
\text { transfer learn- } \\
\text { ing using } \\
\text { MIMIC-III as } \\
\text { source and } \\
\text { UCSF as tar- } \\
\text { get }\end{array}$ & $\begin{array}{l}\text { Carry for- } \\
\text { ward imputa- } \\
\text { tion }\end{array}$ & $\begin{array}{l}\text { Detection with gra- } \\
\text { dient tree boosting: } \\
\text { AUROC }=0.92 \text { for } \\
\text { sepsis; AU- } \\
\text { ROC }=0.87 \text { for se- } \\
\text { vere sepsis at on- } \\
\text { set; AUROC }=0.96 \\
\text { for septic shock } 4 \\
\text { hours before; AU- } \\
\text { ROC }=0.85 \text { for se- } \\
\text { vere sepsis predic- } \\
\text { tion } 4 \text { hours before }\end{array}$ & Not specified & $\begin{array}{l}\text { At onset } \\
\text { of sepsis } \\
\text { and se- } \\
\text { vere sep- } \\
\text { sis; with- } \\
\text { in } 4 \text { hours } \\
\text { preceding } \\
\text { septic } \\
\text { shock and } \\
\text { severe } \\
\text { sepsis }\end{array}$ & $\begin{array}{l}\text { MEWS: AU- } \\
\text { ROC=0.76; } \\
\text { SOFA: AU- } \\
\text { ROC=0.65; } \\
\text { SIRS: AU- } \\
\text { ROC=0.72 }\end{array}$ \\
\hline $\begin{array}{l}\text { Olsen et al, } \\
2018 \text { [46] }\end{array}$ & 178 patients & $\begin{array}{l}160(89.9 \%) \text { had } \\
\geq 1 \text { microevent } \\
\text { occurring during } \\
\text { admission; } 116 \\
\text { patients }(65.2 \%) \\
\text { had } \geq 1 \text { mi- } \\
\text { croevent with a } \\
\text { duration }>15 \\
\text { minutes }\end{array}$ & $\begin{array}{l}\text { Random forest } \\
\text { classifier }\end{array}$ & $\begin{array}{l}\text { Not speci- } \\
\text { fied }\end{array}$ & $\begin{array}{l}\text { Detection of early } \\
\text { signs of deteriora- } \\
\text { tion with random } \\
\text { forest: accura- } \\
\text { cy }=92.2 \% \text {, sensitiv- } \\
\text { ity }=90.6 \% \text {, speci- } \\
\text { ficity }=93.0 \%, \text { AU- } \\
\text { ROC }=96.9 \%\end{array}$ & Not specified & $\begin{array}{l}\text { Not speci- } \\
\text { fied }\end{array}$ & $\begin{array}{l}\text { Compared } \\
\text { with hospital's } \\
\text { current alarm } \\
\text { system: num- } \\
\text { ber of false } \\
\text { alarms de- } \\
\text { creased by } \\
85 \% \text {, number } \\
\text { of missed ear- } \\
\text { ly signs of de- } \\
\text { terioration de- } \\
\text { creased by } \\
73 \%\end{array}$ \\
\hline
\end{tabular}




\begin{tabular}{|c|c|c|c|c|c|c|c|c|}
\hline Study & Cohort & Event rate & ML model(s) & $\begin{array}{l}\text { Missing data } \\
\text { handling }\end{array}$ & $\begin{array}{l}\text { Best ML model } \\
\text { performance }\end{array}$ & $\begin{array}{l}\text { ML model com- } \\
\text { parisons }\end{array}$ & $\begin{array}{l}\text { Predic- } \\
\text { tion win- } \\
\text { dow }\end{array}$ & $\begin{array}{l}\text { Aggregate } \\
\text { weighted } \\
\text { EWS }^{\text {a compar- }} \\
\text { isons }\end{array}$ \\
\hline $\begin{array}{l}\text { Shashikumar } \\
\text { et al, } 2017 \\
{[40]}\end{array}$ & $\begin{array}{l}\text { Patients with } \\
\text { unselected } \\
\text { mixed surgi- } \\
\text { cal proce- } \\
\text { dures }\end{array}$ & 242 sepsis cases & $\begin{array}{l}\text { Elastic net lo- } \\
\text { gistic classifi- } \\
\text { er }\end{array}$ & $\begin{array}{l}\text { Median val- } \\
\text { ues (if multi- } \\
\text { ple measure- } \\
\text { ment were } \\
\text { available); } \\
\text { otherwise, } \\
\text { the old val- } \\
\text { ues were } \\
\text { kept (sam- } \\
\text { ple-and-hold } \\
\text { interpola- } \\
\text { tion); mean } \\
\text { imputation } \\
\text { for replacing } \\
\text { all remaining } \\
\text { missing val- } \\
\text { ues }\end{array}$ & $\begin{array}{l}\text { Event prediction: } \\
\text { elastic net logistic } \\
\text { classifier using en- } \\
\text { tropy features } \\
\text { alone, AU- } \\
\text { ROC }=0.67 \text {, accura- } \\
\text { cy=47\%; elastic } \\
\text { net logistic classifi- } \\
\text { er using social de- } \\
\text { mographics + } \\
\text { EMR }{ }^{\mathrm{y}} \text { features, } \\
\text { AUROC }=0.7 \text {, accu- } \\
\text { racy }=50 \% \text {; elastic } \\
\text { net logistic classifi- } \\
\text { er using all fea- } \\
\text { tures, AU- } \\
\text { ROC }=0.78 \text {, accura- } \\
\text { cy }=61 \%\end{array}$ & Not specified & $\begin{array}{l}4 \text { hours } \\
\text { prior to } \\
\text { onset }\end{array}$ & $\begin{array}{l}\text { No compari- } \\
\text { son }\end{array}$ \\
\hline $\begin{array}{l}\text { Tarassenko } \\
\text { et al, } 2006 \\
{[32]}\end{array}$ & $\begin{array}{l}150 \text { general- } \\
\text { ward pa- } \\
\text { tients }\end{array}$ & Not specified & $\begin{array}{l}\text { Biosign; data } \\
\text { fusion } \\
\text { method: proba- } \\
\text { bilistic model } \\
\text { of normality } \\
\text { in five dimen- } \\
\text { sions }\end{array}$ & $\begin{array}{l}\text { Historic, me- } \\
\text { dian filtering }\end{array}$ & $\begin{array}{l}95 \% \text { of Biosign } \\
\text { alerts were classi- } \\
\text { fied as "True" by } \\
\text { clinical experts }\end{array}$ & Not specified & $\begin{array}{l}\text { Within } \\
120 \text { min- } \\
\text { utes of } \\
\text { event }\end{array}$ & $\begin{array}{l}\text { No compari- } \\
\text { son }\end{array}$ \\
\hline $\begin{array}{l}\text { Van Wyk et } \\
\text { al, } 2017 \text { [33] }\end{array}$ & $\begin{array}{l}2995 \text { pa- } \\
\text { tients }\end{array}$ & $\begin{array}{l}343 \text { patients } \\
(11.5 \%) \text { diag- } \\
\text { nosed with sepsis }\end{array}$ & $\begin{array}{l}\mathrm{CNN}^{\mathrm{Z}} \text { (con- } \\
\text { structed im- } \\
\text { ages using raw } \\
\text { patient data) } \\
\text { with random } \\
\text { dropout to re- } \\
\text { duce overfit- } \\
\text { ting; multilay- } \\
\text { er perceptron } \\
\text { with random } \\
\text { dropout be- } \\
\text { tween layers } \\
\text { to avoid over- } \\
\text { fitting }\end{array}$ & $\begin{array}{l}\text { Not speci- } \\
\text { fied }\end{array}$ & $\begin{array}{l}\text { Event classifica- } \\
\text { tion with a } 1- \\
\text { minute observation } \\
\text { frequency: } \mathrm{CNN} \text {, } \\
\text { accuracy=86.1\%; } \\
\text { event classification } \\
\text { with a } 10 \text {-minute } \\
\text { observation fre- } \\
\text { quency: } \mathrm{CNN} \text {, ac- } \\
\text { curacy }=78.2 \%\end{array}$ & $\begin{array}{l}\text { Event classifica- } \\
\text { tion with a 1- } \\
\text { minute observa- } \\
\text { tion frequency: } \\
\text { multilayer percep- } \\
\text { tron, accura- } \\
\text { cy=76.5\%; } \\
\text { event classifica- } \\
\text { tion with a 10- } \\
\text { minute observa- } \\
\text { tion frequency: } \\
\text { multilayer percep- } \\
\text { tron, accura- } \\
\text { cy=71\% }\end{array}$ & $\begin{array}{l}\text { Not speci- } \\
\text { fied }\end{array}$ & $\begin{array}{l}\text { No compari- } \\
\text { son }\end{array}$ \\
\hline
\end{tabular}




\begin{tabular}{|c|c|c|c|c|c|c|c|c|}
\hline Study & Cohort & Event rate & ML model(s) & $\begin{array}{l}\text { Missing data } \\
\text { handling }\end{array}$ & $\begin{array}{l}\text { Best ML model } \\
\text { performance }\end{array}$ & $\begin{array}{l}\text { ML model com- } \\
\text { parisons }\end{array}$ & $\begin{array}{l}\text { Predic- } \\
\text { tion win- } \\
\text { dow }\end{array}$ & $\begin{array}{l}\text { Aggregate } \\
\text { weighted } \\
\text { EWS }^{\text {a compar- }} \\
\text { isons }\end{array}$ \\
\hline $\begin{array}{l}\text { Yoon et al, } \\
2019 \text { [41] }\end{array}$ & $\begin{array}{l}2809 \text { sub- } \\
\text { jects }\end{array}$ & $\begin{array}{l}787 \text { tachycardia } \\
\text { episodes }\end{array}$ & $\begin{array}{l}\text { Regularized } \\
\text { logistic regres- } \\
\text { sion and ran- } \\
\text { dom forest } \\
\text { classifiers }\end{array}$ & $\begin{array}{l}\text { Discrete } \\
\text { Fourier } \\
\text { transform, } \\
\text { cubic-spline } \\
\text { interpolation } \\
\text { of heart rate } \\
\text { and respirato- } \\
\text { ry rate data } \\
\text { for missing } \\
\text { data as long } \\
\text { as } \geq 20 \% \text { of } \\
\text { the data } \\
\text { were avail- } \\
\text { able }\end{array}$ & $\begin{array}{l}\text { Event prediction: } \\
\text { random forest, } \\
\mathrm{AUC}=0.869, \text { accu- } \\
\text { racy }=0.806\end{array}$ & $\begin{array}{l}\text { Logistic regres- } \\
\text { sion with L1 regu- } \\
\text { larization, } \\
\text { AUC }=0.8284, \text { ac- } \\
\text { curacy }=0.7668\end{array}$ & $\begin{array}{l}\text { Within } 3 \\
\text { hours pre- } \\
\text { ceding } \\
\text { onset }\end{array}$ & $\begin{array}{l}\text { No compari- } \\
\text { son }\end{array}$ \\
\hline
\end{tabular}

${ }^{\mathrm{a}}$ EWS: early warning system.

${ }^{\mathrm{b}} \mathrm{ICU}$ : intensive care unit.

${ }^{\mathrm{c}}$ AUROC: area under the receiver operator characteristic.

${ }^{\mathrm{d}}$ NEWS: National Early Warning Score.

${ }^{\mathrm{e}} \mathrm{CRI}$ : cardiorespiratory instability.

${ }^{\mathrm{f}} \mathrm{AUC}$ : area under the curve.

gPP: positive predictive value.

${ }^{\mathrm{h}}$ SVM: support vector machine.

${ }^{\mathrm{i}}$ SEDS: Singapore Emergency Department Sepsis.

$\mathrm{j}_{\mathrm{qSOFA}}$ : quick Sequential Organ Failure Assessment.

${ }^{k}$ MEWS: Modified Early Warning Score.

${ }^{1}$ APR: area under the precision-recall curve.

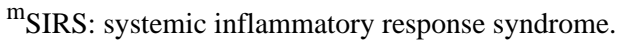

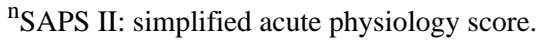

${ }^{\circ}$ PCA: principal component analysis.

PTITA: temporal interval tree association.

${ }^{\mathrm{q}} \mathrm{ANN}$ : artificial neural network.

${ }^{\mathrm{r}}$ LSTM: long short-term memory.

${ }^{\mathrm{s}} \mathrm{RNN}$ : recurrent neural network.

${ }^{\mathrm{t}}$ AUPRC: area under the precision-recall curve.

uTAS: Deep learning-based Triage and Acuity Score.

VTIMI: Thrombolysis in Myocardial Infarction.

${ }^{\mathrm{w}}$ UCSF: University of California, San Francisco.

${ }^{x}$ MIMIC: Medical Information Mart for Intensive Care.

${ }^{\mathrm{y}} \mathrm{EMR}$ : electronic medical record.

${ }^{\mathrm{z}} \mathrm{CNN}$ : convolutional neural network.

\section{Comparison With Aggregate-Weighted EWS}

Nine studies compared the performance of ML-based EWS with aggregate-weighted EWS. Studies exploring cardiorespiratory outcomes, general physiological deterioration, or mortality carried out comparisons with NEWS [42,45], MEWS $[11,26,35,36]$, and the Thrombolysis in Myocardial Infarction score [36]. The 3 studies exploring sepsis-related outcomes additionally included the SOFA, qSOFA, and SIRS criteria and the simplified acute physiology (II) score [23,34,37]. A few studies also drew comparisons with other customized scoring systems individual to their care setting or region such as the Korean Triage and Acuity Score [11], Singapore Emergency
Department Sepsis model [23], and postanesthesia care unit alarm system [46].

In all 9 studies, the ML models performed better than the aggregate-weighted EWS systems for all clinical outcomes except for cardiac arrest in the study by Badriyah et al [45]. For example, in the study by Jang et al [35], a long short-term memory neural network achieved an AUROC of 0.933 , an improvement over MEWS, which achieved an AUROC of 0.886 using the same data. Similarly, in the study by Kwon et al [26], recurrent neural networks achieved an AUROC of 0.85 compared to 0.603 for MEWS and 0.785 for the Korean Triage and Acuity Score. Some studies reported much more modest improvements, such as the study by Chiu et al [42] that achieved 
an AUROC of 0.779 using logistic regression, compared to 0.754 using MEWS for the same 24-hour prediction window. A full side-by-side comparison of ML vs aggregate-weighted EWS is presented in Multimedia Appendix 3.

\section{Discussion}

Based on this scoping review, ML-based EWS models show considerable promise, but there exist several important avenues for future research if these models are to be effectively implemented in clinical practice.

\section{Prediction Window}

A model's prediction window refers to how far in advance a model is predicting an adverse event. Most studies included in our review used a prediction window between 30 minutes [26] and 72 hours [36] before the clinical deterioration took place. The length of a model's prediction window is important because a prediction window that is too short will not yield any real clinical benefit (it would not give a clinical team sufficient time to intervene), but a number of studies $[29,34,37,42]$ showed a decrease in model performance when the prediction window was longer (eg, AUROC drops from 0.88 at the time of onset to 0.74 at 4 hours before the event). Future research seeking to maximize the clinical benefit of ML EWS should strive to achieve an optimum balance between a clinically relevant prediction window and clinically acceptable model performance, rather than simply maximizing a model performance metric, such as AUROC.

\section{Clinically Actionable Explanations}

The studies included in this review focused on ML model development and did not explore how the output of these models would be communicated to clinicians. Since many ML models are "black boxes" [46,47], it may not be immediately clear to clinicians what the likely reason for an alert might be until the patient is assessed, which can cause further delays in time-sensitive scenarios. However, in the broader ML field, there has been significant recent progress in explainable ML techniques, and it has been pointed out that these approaches may be preferred by the medical community and regulators $[48,49]$. Several explanation methods take specific, previously black-box methods, such as convolutional neural networks [50], and allow for post-hoc explanation of their decision-making process. Other explainability algorithms are model-agnostic, meaning they can be applied to any type of model, regardless of its mathematical basis [51]. In the study by Lauritsen et al [52], an explainable EWS was developed based on a temporal convolutional network, using a separate module for explanations. These methodologies are promising, but their application to health care, including to EWS, has been limited. Objective evaluation of the utility of explanation methods is a difficult, ongoing problem, but is an important direction for future research in the area of ML-based EWS if they are to be effectively deployed in clinical practice [53].

\section{Expanded Study Settings}

Nearly all the studies included in this review were conducted in inpatient settings. While EWS are highly valuable in an inpatient context, there is also considerable need in the ambulatory setting, particularly postdischarge. For example, the VISION study [54] found that $1.8 \%$ of all patients die within 30 days postsurgery and $29.4 \%$ of all deaths occurred after patients were discharged from hospital. Patients often receive postoperative monitoring only 3-4 weeks [54] after discharge during a follow-up visit with their surgeon. During this period, it has been shown that many patients suffer from prolonged unidentified hypoxemia [55] and hypotension [56], which are precursors to serious postoperative complications. While EWS research has historically focused on inpatient settings due to the availability of continuous vital signs data, the increasing availability of remote patient monitoring and wearable technologies offer the opportunity to direct future EWS research to the ambulatory setting to address a significant clinical need.

\section{Retrospective Versus Prospective Evaluation}

All but one study [21] included in this review were retrospective in nature, leaving open the possibility that algorithm performance in a clinical environment may be lower than the performance achieved in a controlled retrospective setting [34]. It is also unclear how often these EWS were able to identify clinical deterioration that had not already been detected by a care team. Further, alerts for clinical deterioration may be easily disregarded by clinicians due to alert fatigue, even when the risk of deterioration has been correctly identified [43]. In the single case where an ML-based EWS was studied prospectively, Olsen et al [21] found that the random forest classifier decreased false alarm rates by $85 \%$ and the rate of missed alerts by $73 \%$ when compared to the existing aggregate-weighted alarm system. While the predictions were independently scored for severity by 2 clinician experts, the interpretation of the clinical impact of these alerts was not explored any further, leaving the question of clinical benefit unanswered. Future research into ML-based EWS should begin to include prospective evaluation, both of model accuracy (to understand how model performance is affected when faced with real-world data) and of clinical outcomes (to understand whether alerts in fact produce clinical benefits).

\section{Standardizations of Performance Metrics}

A key observation from this review is the lack of an agreed-upon standard among the research community for reporting performance measures across studies. This makes meaningful comparison between the outcomes of these studies difficult, and where there is overlap, it is not clear that the most clinically relevant metrics have been chosen. The majority of the studies in this review report the AUROC as the main performance metric, reflecting a common practice in the ML literature. However, AUROC may not be adequate for evaluating the performance of the EWS in a clinical setting [57].

As Romero-Brufau et al [58] discussed in their article, AUROC does not incorporate information about the prevalence of physiological deterioration, which can be lower than 0.02 daily in a general inpatient setting. This can make AUROC a misleading metric, leading to overestimation of clinical benefit and underestimation of clinical workload and resources. [58] When the prevalence is low $(<0.1)$, even a model with high sensitivity and specificity may not yield a high posttest probability for a positive prediction [15]. Therefore, reporting 
metrics that incorporate the prevalence would be more appropriate.

The performance of an EWS depends on the tradeoff between 2 goals: early detection of outcomes versus issuance of fewer false-positive alerts to prevent alarm fatigue [43]. Sensitivity can be a good metric to evaluate the first goal as it would provide the percentage of true-positive predictions within a certain time period. To evaluate the clinical burden of false-positive alerts, the positive predictive value, which incorporates prevalence, can be used as it gives a percentage of useful alerts that lead to a clinical outcome. The number needed to evaluate can be a useful measure of clinical utility and cost-efficiency of each alert as it provides the number of patients that need to be evaluated further to detect one outcome. Using these metrics to evaluate tradeoffs between outcome detection and workload would be essential for determining the clinical utility of the EWS [58]. Additionally, the F1 score can also be a useful metric as it provides a measure of the model's overall accuracy through the calculation of the harmonic mean of the precision and recall (sensitivity). Balancing the use of these 2 metrics could yield a more realistic measure of the model's performance [58].

\section{Comparison to "Gold Standard" EWS}

On a related note, only 9 of the studies included in our review made comparisons between their ML-based models and a "gold standard" aggregate-weighted EWS, such as MEWS or NEWS. Future research in the area should report a commonly used aggregate-weighted EWS as a baseline model, which would aid in making effective comparisons between them. NEWS may be particularly well suited to this area of research as its input variables can all be measured automatically and continuously via devices.

\section{Strengths of the Review}

The search strategy was comprehensive while not being too focused on specific clinical outcomes, sampling frequencies, or filtering for time. This allowed for the identification of as many studies as possible that examined the use of ML models and vital signs to predict the risk of patient deterioration. No additional studies were identified through citation tracking after the original search, indicating our search strategy was comprehensive. Unlike previous reviews, inclusion criteria for the review supported the examination of findings from studies conducted across a variety of clinical settings including specialty units or wards and ambulatory care. This helped in characterizing the use of ML-based prediction models in different patient-care environments with varying clinical endpoints. Wherever the original studies provided the data, comparisons were drawn between the performance of the ML models and that of aggregate-weighted EWS. This gives an indication of the differences in accuracy of the models in predicting clinical deterioration.

\section{Limitations}

The findings within this review are subject to some limitations. First, the literature search, assessment of eligibility of full-text articles, inclusion in the review, and extraction of study data were carried out by only 1 author. Second, only the findings from published studies were included in this scoping review, which may affect the results due to publication bias. While studies from a variety of settings were included, the generalizability of our findings may be limited due to the heterogeneity of patient populations, clinical practices, and study methodologies. Sampling procedures and frequencies varied across studies from single to multiple observations of patient vital signs, and clinical outcome definitions were based on different criteria or aggregate-weighted EWS. Finally, due to this variation in ML methods, prediction windows, and outcome reporting, a meta-analysis was not feasible.

\section{Conclusion}

Our findings suggest that ML-based EWS models incorporating easily accessible vital sign measurements are effective in predicting physiological deterioration in patients. Improved prediction performance was also observed with these models when compared to traditional aggregate-based risk stratification tools. The clinical impact of these ML-based EWS could be significant for clinical staff and patients due to decreased false alerts and increased early detection of warning signs for timely intervention, though further development of these models is needed and the necessary prospective research to establish actual clinical utility does not yet exist.

\section{Authors' Contributions}

SM contributed to conceptualization, data collection, data analysis, and manuscript writing. JP contributed to conceptualization, manuscript writing, and manuscript review. WN and SD contributed equally to manuscript writing and review. PD contributed to manuscript writing and review. MM and NB contributed to manuscript review.

\section{Conflicts of Interest}

PJD is a member of a research group with a policy of not accepting honorariums or other payments from industry for their own personal financial gain. They do accept honorariums/payments from industry to support research endeavours and costs to participate in meetings.

Based on study questions PJD has originated and grants he has written, he has received grants from Abbott Diagnostics, AstraZeneca, Bayer, Boehringer Ingelheim, Bristol-Myers-Squibb, Coviden, Octapharma, Philips Healthcare, Roche Diagnostics, Siemens, and Stryker.

PJD has participated in advisory board meetings for GlaxoSmithKline, Boehringer Ingelheim, Bayer, and Quidel Canada. He also attended an expert panel meeting with AstraZeneca and Boehringer Ingelheim.

The other authors declare no conflicts of interest. 


\section{Multimedia Appendix 1}

Search terms.

[DOCX File, 12 KB-Multimedia Appendix 1]

\section{Multimedia Appendix 2}

Description of ML methods and relevant terms.

[DOCX File, 15 KB-Multimedia Appendix 2]

\section{Multimedia Appendix 3}

Comparison between performance of ML based EWS and aggregate EWS.

[DOCX File, $20 \mathrm{~KB}-$ Multimedia Appendix 3]

\section{References}

1. Barfod C, Lauritzen MMP, Danker JK, Sölétormos G, Forberg JL, Berlac PA, et al. Abnormal vital signs are strong predictors for intensive care unit admission and in-hospital mortality in adults triaged in the emergency department - a prospective cohort study. Scand J Trauma Resusc Emerg Med 2012 Apr 10;20:28 [FREE Full text] [doi: 10.1186/1757-7241-20-28] [Medline: 22490208]

2. Hillman KM, Bristow PJ, Chey T, Daffurn K, Jacques T, Norman SL, et al. Antecedents to hospital deaths. Intern Med J 2001 Aug;31(6):343-348. [doi: 10.1046/j.1445-5994.2001.00077.x] [Medline: 11529588 ]

3. McGaughey J, Alderdice F, Fowler R, Kapila A, Mayhew A, Moutray M. Outreach and Early Warning Systems (EWS) for the prevention of intensive care admission and death of critically ill adult patients on general hospital wards. Cochrane Database Syst Rev 2007 Jul 18(3):CD005529. [doi: 10.1002/14651858.CD005529.pub2] [Medline: 17636805]

4. Smith GB, Prytherch DR, Schmidt PE, Featherstone PI, Higgins B. A review, and performance evaluation, of single-parameter "track and trigger" systems. Resuscitation 2008 Oct;79(1):11-21. [doi: 10.1016/j.resuscitation.2008.05.004] [Medline: 18620794]

5. Gardner-Thorpe J, Love N, Wrightson J, Walsh S, Keeling N. The value of Modified Early Warning Score (MEWS) in surgical in-patients: a prospective observational study. Ann R Coll Surg Engl 2006 Oct;88(6):571-575 [FREE Full text] [doi: 10.1308/003588406X130615] [Medline: 17059720]

6. Gao H, McDonnell A, Harrison DA, Moore T, Adam S, Daly K, et al. Systematic review and evaluation of physiological track and trigger warning systems for identifying at-risk patients on the ward. Intensive Care Med 2007 Apr;33(4):667-679. [doi: 10.1007/s00134-007-0532-3] [Medline: 17318499]

7. Subbe CP, Slater A, Menon D, Gemmell L. Validation of physiological scoring systems in the accident and emergency department. Emerg Med J 2006 Nov;23(11):841-845 [FREE Full text] [doi: 10.1136/emj.2006.035816] [Medline: 17057134]

8. Smith GB, Prytherch DR, Meredith P, Schmidt PE, Featherstone PI. The ability of the National Early Warning Score (NEWS) to discriminate patients at risk of early cardiac arrest, unanticipated intensive care unit admission, and death. Resuscitation 2013 Apr;84(4):465-470. [doi: 10.1016/j.resuscitation.2012.12.016] [Medline: 23295778]

9. Tam B, Xu M, Kwong M, Wardell C, Kwong A, Fox-Robichaud A. The Admission Hamilton Early Warning Score (HEWS) Predicts the Risk of Critical Event during Hospitalization. Can Journ Gen Int Med 2017 Feb 24;11(4):1. [doi:

10.22374/cjgim.v11i4.190]

10. Prytherch DR, Smith GB, Schmidt PE, Featherstone PI. ViEWS--Towards a national early warning score for detecting adult inpatient deterioration. Resuscitation 2010 Aug;81(8):932-937. [doi: 10.1016/j.resuscitation.2010.04.014] [Medline: $\underline{20637974]}$

11. Kwon J, Lee Y, Lee Y, Lee S, Park H, Park J. Validation of deep-learning-based triage and acuity score using a large national dataset. PLoS One 2018;13(10):e0205836 [FRE Full text] [doi: 10.1371/journal.pone.0205836] [Medline: 30321231]

12. Bates DW, Saria S, Ohno-Machado L, Shah A, Escobar G. Big data in health care: using analytics to identify and manage high-risk and high-cost patients. Health Aff (Millwood) 2014 Jul;33(7):1123-1131. [doi: 10.1377/hlthaff.2014.0041] [Medline: 25006137]

13. Churpek MM, Yuen TC, Park SY, Gibbons R, Edelson DP. Using electronic health record data to develop and validate a prediction model for adverse outcomes in the wards*. Crit Care Med 2014 Apr;42(4):841-848 [FREE Full text] [doi: 10.1097/CCM.0000000000000038] [Medline: 24247472]

14. Linnen DT, Escobar GJ, Hu X, Scruth E, Liu V, Stephens C. Statistical Modeling and Aggregate-Weighted Scoring Systems in Prediction of Mortality and ICU Transfer: A Systematic Review. J Hosp Med 2019 Mar;14(3):161-169 [FREE Full text] [doi: 10.12788/jhm.3151] [Medline: $\underline{30811322]}$

15. Brekke IJ, Puntervoll LH, Pedersen PB, Kellett J, Brabrand M. The value of vital sign trends in predicting and monitoring clinical deterioration: A systematic review. PLoS One 2019;14(1):e0210875 [FREE Full text] [doi: 10.1371/journal.pone.0210875] [Medline: 30645637] 
16. Tricco AC, Lillie E, Zarin W, O'Brien KK, Colquhoun H, Levac D, et al. PRISMA Extension for Scoping Reviews (PRISMA-ScR): Checklist and Explanation. Ann Intern Med 2018 Oct 02;169(7):467-473 [FREE Full text] [doi: 10.7326/M18-0850] [Medline: 30178033]

17. Zweig MH, Campbell G. Receiver-operating characteristic (ROC) plots: a fundamental evaluation tool in clinical medicine. Clin Chem 1993 Apr;39(4):561-577. [Medline: 8472349]

18. Moher D, Liberati A, Tetzlaff J, Altman DG, PRISMA Group. Preferred reporting items for systematic reviews and meta-analyses: the PRISMA statement. PLoS Med 2009 Jul 21;6(7):e1000097 [FREE Full text] [doi: 10.1371/journal.pmed.1000097] [Medline: 19621072]

19. Higgins JPT, Green S. Cochrane Handbook for Systematic Reviews of Interventions Version 5.1.0. The Cochrane Collaboration. 2011. URL: www.handbook.cochrane.org [accessed 2021-01-09]

20. Collins GS, Reitsma JB, Altman DG, Moons KGM. Transparent Reporting of a multivariable prediction model for Individual Prognosis Or Diagnosis (TRIPOD): the TRIPOD Statement. Br J Surg 2015 Feb;102(3):148-158. [doi: 10.1002/bjs.9736] [Medline: 25627261]

21. Olsen RM, Aasvang EK, Meyhoff CS, Dissing Sorensen HB. Towards an automated multimodal clinical decision support system at the post anesthesia care unit. Comput Biol Med 2018 Oct 01;101:15-21. [doi: 10.1016/j.compbiomed.2018.07.018] [Medline: 30092398]

22. Larburu N, Artetxe A, Escolar V, Lozano A, Kerexeta J. Artificial Intelligence to Prevent Mobile Heart Failure Patients Decompensation in Real Time: Monitoring-Based Predictive Model. Mobile Information Systems 2018 Nov 05;2018:1-11. [doi: $10.1155 / 2018 / 1546210]$

23. Chiew CJ, Liu N, Tagami T, Wong TH, Koh ZX, Ong MEH. Heart rate variability based machine learning models for risk prediction of suspected sepsis patients in the emergency department. Medicine (Baltimore) 2019 Feb;98(6):e14197 [FREE Full text] [doi: 10.1097/MD.0000000000014197] [Medline: 30732136]

24. Churpek MM, Adhikari R, Edelson DP. The value of vital sign trends for detecting clinical deterioration on the wards. Resuscitation 2016 May;102:1-5 [FREE Full text] [doi: 10.1016/j.resuscitation.2016.02.005] [Medline: 26898412]

25. Clifton L, Clifton DA, Pimentel MAF, Watkinson PJ, Tarassenko L. Predictive monitoring of mobile patients by combining clinical observations with data from wearable sensors. IEEE J Biomed Health Inform 2014 May;18(3):722-730. [doi: 10.1109/JBHI.2013.2293059] [Medline: 24808218]

26. Kwon J, Lee Y, Lee Y, Lee S, Park J. An Algorithm Based on Deep Learning for Predicting In-Hospital Cardiac Arrest. J Am Heart Assoc 2018 Jun 26;7(13):1 [FREE Full text] [doi: 10.1161/JAHA.118.008678] [Medline: 29945914]

27. Forkan ARM, Khalil I. PEACE-Home: Probabilistic estimation of abnormal clinical events using vital sign correlations for reliable home-based monitoring. Pervasive and Mobile Computing 2017 Jul;38:296-311. [doi: 10.1016/j.pmcj.2016.12.009]

28. Forkan ARM, Khalil I. A clinical decision-making mechanism for context-aware and patient-specific remote monitoring systems using the correlations of multiple vital signs. Comput Methods Programs Biomed 2017 Feb;139:1-16. [doi: 10.1016/j.cmpb.2016.10.018] [Medline: 28187881]

29. Forkan ARM, Khalil I, Atiquzzaman M. ViSiBiD: A learning model for early discovery and real-time prediction of severe clinical events using vital signs as big data. Computer Networks 2017 Feb;113:244-257. [doi: 10.1016/j.comnet.2016.12.019]

30. Lee J, Scott D, Villarroel M, Clifford G, Saeed M, Mark R. Open-access MIMIC-II database for intensive care research. Conf Proc IEEE Eng Med Biol Soc 2011;2011:8315-8318. [doi: 10.1109/iembs.2011.6092050] [Medline: 22256274]

31. Johnson AEW, Pollard TJ, Shen L, Lehman LH, Feng M, Ghassemi M, et al. MIMIC-III, a freely accessible critical care database. Sci Data 2016 May 24;3:160035 [FREE Full text] [doi: 10.1038/sdata.2016.35] [Medline: 27219127]

32. Tarassenko L, Hann A, Young D. Integrated monitoring and analysis for early warning of patient deterioration. Br J Anaesth 2006 Jul;97(1):64-68 [FREE Full text] [doi: 10.1093/bja/ael113] [Medline: 16707529]

33. van Wyk F, Khojandi A, Kamaleswaran R, Akbilgic O, Nemati S, Davis RL. How much data should we collect? A case study in sepsis detection using deep learning. 2017 Presented at: IEEE Healthcare Innovations and Point of Care Technologies (HI-POCT); November 6-8, 2017; Bethesda, MD. [doi: 10.1109/hic.2017.8227596]

34. Mao Q, Jay M, Hoffman JL, Calvert J, Barton C, Shimabukuro D, et al. Multicentre validation of a sepsis prediction algorithm using only vital sign data in the emergency department, general ward and ICU. BMJ Open 2018 Jan 26;8(1):e017833 [FREE Full text] [doi: 10.1136/bmjopen-2017-017833] [Medline: 29374661]

35. Jang D, Kim J, Jo YH, Lee JH, Hwang JE, Park SM, et al. Developing neural network models for early detection of cardiac arrest in emergency department. Am J Emerg Med 2020 Jan;38(1):43-49. [doi: 10.1016/j.ajem.2019.04.006] [Medline: $\underline{30982559]}$

36. Liu N, Koh ZX, Goh J, Lin Z, Haaland B, Ting BP, et al. Prediction of adverse cardiac events in emergency department patients with chest pain using machine learning for variable selection. BMC Med Inform Decis Mak 2014 Aug 23;14:75 [FREE Full text] [doi: 10.1186/1472-6947-14-75] [Medline: 25150702]

37. Desautels T, Calvert J, Hoffman J, Jay M, Kerem Y, Shieh L, et al. Prediction of Sepsis in the Intensive Care Unit With Minimal Electronic Health Record Data: A Machine Learning Approach. JMIR Med Inform 2016 Sep 30;4(3):e28 [FREE Full text] [doi: 10.2196/medinform.5909] [Medline: 27694098]

38. Ho J, Park Y. Learning from different perspectives: Robust cardiac arrest prediction via temporal transfer learning. Annu Int Conf IEEE Eng Med Biol Soc 2017 Jul;2017:1672-1675. [doi: 10.1109/EMBC.2017.8037162] [Medline: 29060206] 
39. Li X, Wang Y. Adaptive online monitoring for ICU patients by combining just-in-time learning and principal component analysis. J Clin Monit Comput 2016 Dec;30(6):807-820. [doi: 10.1007/s10877-015-9778-4] [Medline: 26392184]

40. Shashikumar SP, Stanley MD, Sadiq I, Li Q, Holder A, Clifford GD, et al. Early sepsis detection in critical care patients using multiscale blood pressure and heart rate dynamics. J Electrocardiol 2017;50(6):739-743 [FREE Full text] [doi: 10.1016/j.jelectrocard.2017.08.013] [Medline: 28916175]

41. Yoon JH, Mu L, Chen L, Dubrawski A, Hravnak M, Pinsky MR, et al. Predicting tachycardia as a surrogate for instability in the intensive care unit. J Clin Monit Comput 2019 Dec;33(6):973-985 [FREE Full text] [doi: 10.1007/s10877-019-00277-0] [Medline: $\underline{30767136]}$

42. Chiu Y, Villar SS, Brand JW, Patteril MV, Morrice DJ, Clayton J, et al. Logistic early warning scores to predict death, cardiac arrest or unplanned intensive care unit re-admission after cardiac surgery. Anaesthesia 2020 Feb;75(2):162-170 [FREE Full text] [doi: 10.1111/anae.14755] [Medline: $\underline{\text { 31270799] }}$

43. Guillame-Bert M, Dubrawski A, Wang D, Hravnak M, Clermont G, Pinsky MR. Learning temporal rules to forecast instability in continuously monitored patients. J Am Med Inform Assoc 2017 Jan;24(1):47-53 [FREE Full text] [doi: 10.1093/jamia/ocw048] [Medline: 27274020]

44. Chen L, Ogundele O, Clermont G, Hravnak M, Pinsky MR, Dubrawski AW. Dynamic and Personalized Risk Forecast in Step-Down Units. Implications for Monitoring Paradigms. Ann Am Thorac Soc 2017 Mar;14(3):384-391 [FREE Full text] [doi: 10.1513/AnnalsATS.201611-9050C] [Medline: 28033032]

45. Badriyah T, Briggs JS, Meredith P, Jarvis SW, Schmidt PE, Featherstone PI, et al. Decision-tree early warning score (DTEWS) validates the design of the National Early Warning Score (NEWS). Resuscitation 2014 Mar;85(3):418-423. [doi: 10.1016/j.resuscitation.2013.12.011] [Medline: 24361673]

46. Cabitza F, Rasoini R, Gensini GF. Unintended Consequences of Machine Learning in Medicine. JAMA 2017 Aug 08;318(6):517-518. [doi: 10.1001/jama.2017.7797] [Medline: 28727867]

47. Stead WW. Clinical Implications and Challenges of Artificial Intelligence and Deep Learning. JAMA 2018 Sep 18;320(11):1107-1108. [doi: 10.1001/jama.2018.11029] [Medline: $\underline{\text { 30178025] }}$

48. Tonekaboni S, Joshi S, McCradden MD, Goldenberg A. What Clinicians Want: Contextualizing Explainable Machine Learning for Clinical End Use. Proceedings of the 4th Machine Learning for Healthcare Conference 2019;106:359-380.

49. Wiens J, Saria S, Sendak M, Ghassemi M, Liu VX, Doshi-Velez F, et al. Do no harm: a roadmap for responsible machine learning for health care. Nat Med 2019 Sep;25(9):1337-1340. [doi: 10.1038/s41591-019-0548-6] [Medline: 31427808]

50. Wang ZJ, Turko R, Shaikh O, Park H, Das N, Hohman F, et al. CNN EXPLAINER: Learning Convolutional Neural Networks with Interactive Visualization. IEEE Trans. Visual. Comput. Graphics 2020:1. [doi: 10.1109/tvcg.2020.3030418]

51. Ribeiro MT, Singh S, Guestrin C. "Why Should I Trust You?": Explaining the Predictions of Any Classifier. Cornell University. 2016. URL: https://arxiv.org/abs/1602.04938 [accessed 2021-01-09]

52. Lauritsen SM, Kristensen M, Olsen MV, Larsen MS, Lauritsen KM, Jørgensen MJ, et al. Explainable artificial intelligence model to predict acute critical illness from electronic health records. Nat Commun 2020 Jul 31;11(1):3852 [FREE Full text] [doi: 10.1038/s41467-020-17431-x] [Medline: 32737308]

53. Sokol K, Flach P. Explainability Fact Sheets: A Framework for Systematic Assessment of Explainable Approaches. Cornell University. 2019. URL: https://arxiv.org/abs/1912.05100 [accessed 2021-01-09]

54. Vascular Events in Noncardiac Surgery Patients Cohort Evaluation (VISION) Study Investigators, Spence J, LeManach Y, Chan MT, Wang CY, Sigamani A, et al. Association between complications and death within 30 days after noncardiac surgery. CMAJ 2019 Jul 29;191(30):E830-E837 [FREE Full text] [doi: 10.1503/cmaj.190221] [Medline: 31358597]

55. Sun Z, Sessler DI, Dalton JE, Devereaux PJ, Shahinyan A, Naylor AJ, et al. Postoperative Hypoxemia Is Common and Persistent: A Prospective Blinded Observational Study. Anesth Analg 2015 Sep;121(3):709-715 [FREE Full text] [doi: 10.1213/ANE.0000000000000836] [Medline: 26287299]

56. Turan A, Chang C, Cohen B, Saasouh W, Essber H, Yang D, et al. Incidence, Severity, and Detection of Blood Pressure Perturbations after Abdominal Surgery: A Prospective Blinded Observational Study. Anesthesiology 2019 Apr;130(4):550-559 [FREE Full text] [doi: 10.1097/ALN.0000000000002626] [Medline: 30875354]

57. Cook NR. Use and misuse of the receiver operating characteristic curve in risk prediction. Circulation 2007 Feb 20;115(7):928-935 [FREE Full text] [doi: 10.1161/CIRCULATIONAHA.106.672402] [Medline: 17309939]

58. Romero-Brufau S, Huddleston JM, Escobar GJ, Liebow M. Why the C-statistic is not informative to evaluate early warning scores and what metrics to use. Crit Care 2015 Aug 13;19:285 [FREE Full text] [doi: 10.1186/s13054-015-0999-1] [Medline: $\underline{26268570}]$

\section{Abbreviations}

AUROC: area under the receiver operating characteristic

AVPU: alert, verbal, pain, unresponsive

BP: blood pressure

ED: emergency department

EWS: early warning system 
HR: heart rate

ICU: intensive care unit

MEWS: Modified Early Warning Score

MIMIC: Medical Information Mart for Intensive Care

ML: machine learning

NEWS: National Early Warning Score

PRISMA: Preferred Reporting Items for Systematic Reviews and Meta-Analyses

qSOFA: quick Sequential Organ Failure Assessment

RR: respiratory rate

TRIPOD: Transparent Reporting of a Multivariable Prediction Model for Individual Prognosis or Diagnosis

Edited by G Eysenbach; submitted 21.10.20; peer-reviewed by N Liu, J Kellett; comments to author 07.11.20; revised version received 19.12.20; accepted 20.12.20; published 04.02.21

Please cite as:

Muralitharan S, Nelson W, Di S, McGillion M, Devereaux PJ, Barr NG, Petch J

Machine Learning-Based Early Warning Systems for Clinical Deterioration: Systematic Scoping Review

J Med Internet Res 2021;23(2):e25187

URL: https://www.jmir.org/2021/2/e25187

doi: $10.2196 / 25187$

PMID: 33538696

(CSankavi Muralitharan, Walter Nelson, Shuang Di, Michael McGillion, PJ Devereaux, Neil Grant Barr, Jeremy Petch. Originally published in the Journal of Medical Internet Research (http://www.jmir.org), 04.02.2021. This is an open-access article distributed under the terms of the Creative Commons Attribution License (https://creativecommons.org/licenses/by/4.0/), which permits unrestricted use, distribution, and reproduction in any medium, provided the original work, first published in the Journal of Medical Internet Research, is properly cited. The complete bibliographic information, a link to the original publication on http://www.jmir.org/, as well as this copyright and license information must be included. 